\title{
L'HOMME L'Homme
}

171-172 | 2004

Musique et anthropologie

\section{L'efficacité musicale: musiquer pour survivre}

Le cas des Pygmées

\section{Gilbert Rouget}

\section{OpenEdition}

Journals

Édition électronique

URL : http://journals.openedition.org/lhomme/24855

DOI : 10.4000//homme.24855

ISSN : 1953-8103

Éditeur

Éditions de l'EHESS

\section{Édition imprimée}

Date de publication : 1 décembre 2004

Pagination : 27-52

ISSN : 0439-4216

\section{Référence électronique}

Gilbert Rouget, "L'efficacité musicale: musiquer pour survivre », L'Homme [En ligne], 171-172 | 2004, mis en ligne le 01 janvier 2006, consulté le 10 décembre 2020. URL : http://journals.openedition.org/ Ihomme/24855 ; DOI : https://doi.org/10.4000//homme.24855 


\title{
L'efficacité musicale : musiquer pour survivre Le cas des Pygmées
}

\author{
Gilbert Rouget
}

J.

N'APPRENDRAI RIEN à personne en rappelant ici* que les Pygmées ${ }^{1}$, chasseurs-cueilleurs de la grande forêt équatoriale africaine, sont connus pour être des gens qui chantent et dansent - en un mot, qui musiquent ${ }^{* *}$ - beaucoup. Leur musique, essentiellement vocale et polyphonique, a été abondamment enregistrée et reproduite sur disque ; sa description et son analyse musicologique ont fait l'objet de nombreuses publications. Toutes s'accordent à dire qu'une très importante partie du répertoire musical des Pygmées est en étroit rapport avec la chasse, laquelle constitue, ou en tous cas constituait, il y a peu de temps encore - mais plus guère maintenant, car leur mode de vie traditionnel est en pleine et dramatique mutation ${ }^{2}$-, leur principal moyen d'existence. Ces chants, le plus souvent associés à diverses formes de danse, ont (ou avaient) pour objet soit d'assurer directement le succès de la chasse, soit de se concilier la bienveillance des

* Les notes appelées par des chiffres sont renvoyées en fin d'article, p. 43.

** «Musiquer ": dans La Musique et la transe (1980 : 155), j’ai proposé de réhabiliter le terme, au passé plus qu'honorable (Diderot, Rousseau) et très injustement tombé en désuétude, comme convenant particulièrement bien aux besoins de l'ethnomusicologie. À côté de l'expression «faire de la musique", qui met l'accent, qu'on le veuille ou non, sur le produit de l'action musicale, "musiquer » le met sur l'action même, vue indépendamment de son résultat : aux yeux de l'ethnomusicologue la manière de faire de la musique importe tout autant que la musique elle-même. Dans l'édition américaine de ce livre (Music and Trance, 1985 : 103), faute qu'il existe en anglais un verbe intransitif équivalent à celui du français musiquer, un néologisme, to musicate, fut proposé au lecteur, puis utilisé dans la suite de l'ouvrage. Sauf erreur de ma part, le terme n'eut aucun succès en Amérique. Mais quelques années plus tard, en Angleterre, apparut, sous la plume de Christopher Small $(1996,1998)$, celui de musicking, lequel fut abondamment repris peu après, aux États-Unis, par Charles Keil (1998, passim) et semble devenir aujourd'hui d'un usage relativement courant. Il est clair qu'en anglais le besoin se faisait impérieusement sentir d'un vocable en rapport avec l'importance prise en ethnomusicologie par ce que les anglophones nomment performance. Par ailleurs, rappelons ici d'une part l'allemand musizieren, de l'autre l'italien musicare, tous deux traduits par «musiquer ", respectivement dans le Harrap's et dans le Dizionario Maggiore Fr.-It/It.Fr. de Larousse, avec, comme en français, les deux sens de (Rousseau) « faire de la -» 
" esprits $»^{3}$ de la forêt, dispensateurs de toute chose. Bref, si les Pygmées chantent et dansent (ou chantaient et dansaient) ainsi, c'est (c'était) principalement dans une intention propitiatoire, ou sinon conjuratoire. Ils en attendent (en attendaient) que le gibier abonde et que la chasse soit heureuse. Naguère encore celleci l'était la plupart du temps, preuve pour eux que leurs chants et leurs danses étaient sous ce rapport parfaitement efficaces. C'est sur la nature de cette efficacité que l'on voudrait d'abord s'interroger, pour examiner ensuite, d'une manière plus générale, le rôle de la pratique musicale chez ces Pygmées.

Avant d'y venir, retournons un instant à la question du temps. On vient de le dire, le présent des Pygmées n'est plus ce qu'il était. D’où l'emploi alterné des temps présent et imparfait dans le texte qu'on vient de lire : il signale que suivant qu'on se situe dans un temps ou dans l'autre la place que tiennent dans leur vie la chasse et, partant, la musique qui lui correspond, n'est pas la même. Sans pour autant nous détourner de la réalité, si triste, qu'est pour eux le temps présent, dans ce qui suit nous n'aurons plus en vue que celle - bien plus réconfortante, soit dit sans rousseauisme - de leur passé. Pourquoi ? Pour deux raisons. La première parce que c'est celle que j'ai connue - en 1946, il y a cinquante-huit ans, au cours de la mission Ogooué-Congo, chez les BaBinga de la Haute-Sangha ${ }^{4}$, , ce qui me met en droit d'en parler. La seconde parce que, pygmée ou autre, c'est dans tous les cas le passé d'une civilisation qui détermine, dans une grande mesure au moins, son présent et non l'inverse. Dans la problématique qui est ici la nôtre - celle de l'efficacité prêtée en l'occurrence à la musique -, c'est le passé, avec tout ce qu'il en persiste dans le présent, bien sûr, qu'il nous fallait d'abord interroger.

Le lecteur trouvera en note 5 (p. 43) un bref inventaire des enregistrements réalisés en 1946, au cours de la mission dite "Ogooué-Congo », chez les BaBinga dont on vient de parler. Ceux-ci appartenaient à deux groupes distincts, formant

[suite de la note **] (Diderot) "mettre en -» musique. En français, "musiquer », dans le sens qui lui est ici donné, est avec «musique» dans le même rapport que celui qu'ont "parler » et "parole» dans le domaine (si proche) du langage. Employé nominalement «musiquer " permet qu'on parle $\mathrm{du}$ « musiquer » de telle personne ou de tel ou tel groupe, comme on le fait couramment d'un " parler». À l'expression "musicien / musicienne amateur», proposons d'ajouter «musiqueur, musiqueuse", susceptibles des mêmes connotations, louangeuses ou dépréciatives. Rappelons enfin "musiquier", répondant à "parolier", inventé par Boris Vian pour désigner celui qui fait la musique d'une chanson. Musiquer (verbe et nom), musiquier, musiqueur, musiqueuse, musiquant(e), musiqué(e), autant de termes venant élargir le vocabulaire singulièrement restreint dont on dispose pour parler de la musique, de ceux qui en font et de ce qu'on en fait.

Ajoutons que «musiquer » est le plus souvent pris ici dans son sens le plus large, englobant les deux aspects fréquemment inséparables d'une seule et même action (accomplie par une seule et même personne et/ou par un seul et même groupe), consistant à la fois à faire de la musique (vocale et/ou instrumentale) et à danser.

"Qu'est-ce que la musique? » est la question que pose, d'entrée de jeu, l'impressionnant ouvrage collectif récemment paru sous le titre The Origins of Music (Nils L. Wallin et al. 2000: 8). À cette grande interrogation, il n'est guère fourni que de bien décevantes réponses. Peut-être eût-il fallu se demander, plus qu'on ne l'a fait, «qu'est-ce que musiquer? " Ou encore, partant non du produit mais de la production de l'activité musicale, poser la question non des origines, mais des fondements de la musique. 
deux campements différents, celui des BaMbenzélé d'une part, celui des BaNgombé de l'autre. Ces enregistrements ont fait l'objet, à l'époque, de diverses publications (cf. Roche et Rouget dans la bibliographie et la discographie de cet article). Bien que les travaux ultérieurs sur la musique des Pygmées - ceux de Colin Turnbull, de Simha Arom, de Pierre Sallée et de Michelle Kisliuk, pour ne citer que les plus connus de ceux auxquels nous aurons à nous référer - s'étendent à présent sur un demi-siècle, et que depuis 1946 les conditions d'existence de ce peuple aient beaucoup changé, nous cesserons, sauf cas particulier, d'en tenir compte et de spécifier s'il s'agit, dans notre discours, de l'actuel ou du révolu, pour les confondre tous deux dans une sorte de présent intemporel, qui n'est évidemment plus celui d'aujourd'hui.

\section{Trois actions musicales menées en vue du succès de la chasse}

Examinons les relations que font les Pygmées entre leur pratique de la musique et celle de la chasse, en partant de données fournies par trois actions musicales observées durant la mission Ogooué-Congo. Toutes trois sont extraites du répertoire d'un seul de nos deux groupes BaBinga, celui des BaNgombé, réputés pour être des chasseurs d'éléphant (chasse à la sagaie), à la différence des BaMbenzélé qui ne chassaient plus qu'au filet. Pourquoi ces trois-là ? Parce qu'elles m’ont été présentées, expressément et de plusieurs sources, comme visant à favoriser la rencontre de la "viande » et l'heureuse issue de la chasse. Ce sont:

- une grande action chorégraphico-musicale dite Edzingi, du nom du personnage masqué qui en est le héros principal et qui incarne un "esprit » de la forêt, figure centrale des croyances religieuses de ces Pygmées ;

- une danse des chasseurs, dite bukela;

- une pièce chorale de femmes, dite yeli.

[N. B. Edzingi et yeli: des enregistrements de 1946 en sont reproduits sur le $\mathrm{CD}$ encarté en fin de volume \# I à 3.]

$E d z i n g i^{6}$ est un rituel qui doit impérativement s'exécuter après le retour d'une chasse couronnée par la mort d'un éléphant mâle porteur de grandes défenses. Il s'agit d'un spectacle auquel participe le campement tout entier, mais dont le principal protagoniste est, précisément, Edzingi. Surgi de la forêt et représenté par un personnage masqué disparaissant entièrement sous un costume fait de plusieurs étages superposés de raphia frais, Edzingi vient tournoyer sur la place de danse, alternativement dressé ou tassé sur lui-même, entouré de près par trois ou quatre Pygmées qui dirigent plus ou moins étroitement ses évolutions. Groupées un peu à l'écart, les femmes chantent presque continûment, mêlant leurs parties chorales à celles des hommes qui accompagnent le masque en dansant plus ou moins près de lui (cf. la suite des planches hors-texte). Après plusieurs heures coupées d'interruptions plus ou moins brèves, pour finir, au terme d'une de ses évolutions, Edzingi reste longuement à terre, cependant que le chœur entonne une psalmodie jusqualors jamais entendue (cf. CD \# I). Il y a toutes les raisons de penser que 
ce déroulement du spectacle n'est rien d'autre qu'une figuration de la chasse à l'éléphant qui vient d'avoir lieu, le comportement d'Edzingi visant à représenter celui de l'animal blessé puis succombant, en vue de conjurer les effets néfastes de sa mise à mort, le rituel ayant pour but, m'a-t-on dit, de garantir le succès des chasses à venir. Tel que nous l'avons vu, ce théâtre, car c'en est un, et de très haute tenue - réalisation, en quelque manière et à l'échelle de cette minuscule communauté, de l'« œuvre d'art totale »(Gesamtkunstwerke) rêvée par Wagner-, s'est répété deux jours de suite.

Bukela ${ }^{7}$ est une danse qui, comme Edzingi, mobilise tout le campement, femmes et hommes, mais cette fois avant le départ de ceux-ci pour une expédition de chasse au phacochère ou à l'éléphant. Elle a lieu le plus souvent de nuit, autour du feu entretenu au centre du campement mué en place de danse. Assises par terre, les femmes forment un petit groupe compact qui chante continûment, en battant des mains. L'une d'elles frappe une cloche de fer plantée en terre. Deux tambours, battus par deux jeunes hommes, accompagnent presque sans arrêt le chant, auquel participent les chasseurs qui, eux seuls, dansent. Disposés en file les uns derrière les autres, ceux-ci se déplacent en décrivant longuement cercles après cercles autour du feu. La danse consiste à avancer en marchant de manière comme saccadée au rythme des tambours, pieds alternativement haut levés et posés bien à plat sur le sol, genoux à demi fléchis, buste et nuque raides. Nul bonheur, nulle gaieté. Totalement absorbés par ce qu'ils font, semblables à des automates, les danseurs donnent l'impression d'être douloureusement ailleurs. De temps à autre, sur un ordre de celui qui marche en tête et qui est le chef à la fois de la danse et de la chasse, tous s'arrêtent et, tournés vers l'intérieur du cercle, font mine d'examiner soigneusement le sol. Le moment venu, sur un autre ordre, ils reprennent imperturbablement leur pérégrination, les deux figures de danse alternant de la sorte aussi longtemps qu'il le faut, autrement dit le temps nécessaire à la découverte sur le sol de traces annonçant la présence du gibier recherché et préfigurant l'heureuse issue de la chasse. Comme on voit, bukela n'est manifestement rien d'autre que le déroulement d'une action magique menée suivant le principe bien connu (Marcel Mauss) qui veut que le semblable entraîne le semblable.

$Y e l i{ }^{8}$ est tout autre chose. À l'inverse des deux actions précédentes, ce n'est ni une danse, ni une affaire d'hommes. Le mot désigne un certain chant choral, polyphonique bien sûr, et tout particulièrement jodlé ${ }^{9}$, exécuté par un petit groupe de femmes en vue du succès de la chasse à l'éléphant à laquelle leurs hommes se préparent à partir. La chanteuse principale, celle qui dirige le chœur et dont dépend par conséquent la qualité de son exécution, est la femme du chef des chasseurs, ce qui est de toute évidence significatif. Ce l'est d'autant plus sûrement que, signalons-le ici, tout au long du mois précédant leur départ, ceux-ci doivent observer un rigoureux interdit sexuel ${ }^{10}$. Dans son article "Chasse, sexualité et musique ", consacré à l'arc musical des femmes pygmées, Vincent Dehoux (1995: 80-81) propose une interprétation argumentée de cette abstinence et du pourquoi de ses heureux effets sur l'issue de la chasse à l'éléphant. Chanté, trois jours de suite, avant le départ ${ }^{11}$ des hommes pour cette chasse, yeli, m’ont dit les 
BaNgombé de la manière la plus formelle et à plusieurs reprises, fera que les chasseurs trouveront « la viande ". "Les femmes font mulumbi yeli, alors les éléphants et les cochons viennent tout près", me dit le chef du village ngoundi de Gandicolo, dont dépendaient ces Pygmées. «Chant magique » c'est ainsi que me le présenta spontanément mon interprète ${ }^{12}$, qui n'était pas ethnographe et n'avait pas lu Marcel Mauss, mais n'en savait pas moins, aux nuances près, ce qu'il disait.

\section{Croyances : l'efficacité musicale symbolique}

Nul ne contestera que si la magie ${ }^{13}$ et la religion (parfois difficiles à distinguer l'une de l'autre) fonctionnent, c'est parce qu'on y croit. À quelle catégorie de croyance nos trois actions musicales doivent-elles, dans l'esprit des BaBinga, leur efficacité?

La première, Edzingi, s'inscrit dans le droit fil des croyances religieuses. Rendu propice par une représentation théâtrale entièrement faite de chants et de danses, l'« esprit » que désigne ce nom assurera le succès de la chasse. De quelle manière? C'est son affaire et l'on s'en remet à lui. Nous sommes bien là dans la religion.

La deuxième, bukela, la danse des chasseurs, totalement différente de la première, mais comme elle action chorégraphico-musicale, relève d'un tout autre ordre de croyance. Le simulacre d'une chasse menée à bonne fin assure le succès de l'authentique chasse à venir. Le semblable entrâne le semblable. Nous sommes bien là dans la magie.

La troisième, yeli, opère d'une autre manière que les deux précédentes. Laquelle? Par le pouvoir du chant, bien évidemment, et de lui seul, puisqu'entièrement vocalisé il ne comporte pas de parole et que, par ailleurs, il n'est aucunement dansé. Mais quel pouvoir, ou quelle symbolique, bref quel rapport yeli a-t-il avec la chasse? Ce chant n'en imite aucun aspect, ni chorégraphique, vienton de voir, ni sonore, car contrairement à la chasse au filet qui, à un certain moment, se fait à grand renfort de cris, la chasse à l'éléphant se mène dans le plus grand silence. Le charme - carmen - de ce chant réside par conséquent ailleurs. Où ? Dans sa globalité, selon toute apparence, ou, si l'on préfere, dans sa structure, laquelle consiste d'une part à présenter la forme la plus achevée de l'art vocal des Pygmées, mariant le jodel (dérivé ou en tout cas proche parent du cri de chasse) et la polyphonie, de l'autre, rappelons-le, à être le fait d'un petit groupe de femmes, exclusivement femmes de chasseurs, animé, sinon dirigé par celle d'entre elles qui est la femme de leur chef.

Il y a, peut-on voir, homologie entre les deux actions: les femmes chantent comme leurs hommes chassent, mais qu'elle soit ou non significative, c'est en fait sur une certaine qualité du son que se fonde la symbolique de yeli. Nom commun, yeli, ou plus couramment yeyi (Thompson 1991: 51-52) ou yei (Arom $1991: 27$, n. 3), est le mot qui désigne, chez les Pygmées du grand groupe aka, le chant jodlé ${ }^{14}$. Robert Farris Thompson insiste à plusieurs reprises sur le contenu sensible, émotionnel et affectif du yeyi. Une femme biyaka, écrit-il (1991 : 50), lui a expliqué : «nous jodlons dans la forêt pour manger. Le jodel 
fait plaisir aux esprits de la forêt et alors ils nous donnent du gibier ». De son côté, Pierre Sallée écrivait en 1975, à propos des mêmes Pygmées, mais sans parler explicitement de yeli : "leurs polyphonies vocales les mettent en communication avec la forêt qui en renvoyant l'écho les met en relation avec les êtres étranges qui la peuplent». C'est manifestement dans cette perspective, religieuse ou magico-religieuse, qu'il convient de situer la croyance des Pygmées BaNgombé dans l'efficacité du yeli très particulier qu'on vient de décrire.

Notons ici que la situation est la même chez les Pygmées non plus de l'Ouest de la forêt équatoriale mais de l'Est, ceux de l'Itouri, les BaMbuti. « Par leur acte de chanter les chants sacrés [du molimo, leur grand rituel dont nous parlerons plus loin], ils communient avec le dieu de la forêt. Celui-ci les entendra, alors qu'il ignore leurs chants ordinaires ", écrit Colin Turnbull (CD 1991 : 2-3). C'est donc bien par la médiation d'un esprit ou d'un génie de la forêt qu'ici encore le chant opère. Mais, précise Colin Turnbull, pour ces chants, qui se réduisent la plupart du temps à "des suites de voyelles " - ce qui est le cas, précisément, du yeli des BaNgombé qui présentement nous occupe -, c'est « le son (the sound) » qui constitue la "véritable signification du chant, son importance et son pouvoir". Ce «son", quel est-il au juste? Colin Turnbull n'en dit rien. Mais l'écoute du "Molimo Song of Devotion to the Forest", plage $26 \mathrm{du}$ CD Mbuti Pygmies of the Ituri Rainforest (1991) nous renseigne parfaitement sur ce point: ce son, c'est le jodel. À ceci près qu'il n'est exécuté que par des hommes, ce "chant de dévotion " a la même composition que yeli: voix soliste caractérisée par son emploi systématique et spectaculaire du jodel, concertant avec un chœur polyphonique.

Cela étant, il n'est guère aventureux de penser que pour les Pygmées le jodel est en quelque sorte le langage que comprennent les dieux. Pourquoi ? Manifestement parce que dans cette acoustique de cathédrale qu'est en fait la forêt, le jodel, si inattendu que ce soit (nous ne sommes pas au Tyrol), sonne particulièrement bien et s'entend de loin. Ce qui explique, d'une part, que les chasseurs y aient recours pour communiquer, de l'autre que, comme le rapporte Colin Turnbull à plusieurs reprises, les chants des Pygmées BaMbuti aient pour objet de "réveiller la forêt ", et qu'enfin, revenons aux BaNgombé, les femmes des chasseurs chantent yeli pour (grâce aux génies de la forêt) faire que la chasse soit heureuse.

Les trois actions musicales menées en vue du succès de la chasse dont on a parlé plus haut sont les seules qu'il m'ait été donné d'observer et d'enregistrer, durant mon court séjour de 1946, chez les BaNgombé, mais ceux-ci en pratiquaient également d'autres, parmi lesquelles les deux qui suivent et qu'il me faut maintenant très brièvement mentionner. En premier lieu, la danse dite djoboko ${ }^{15}$, que je n'ai vue pratiquée que chez leurs voisins BaMbenzélé ; nous y reviendrons plus loin. En second lieu, le rituel chorégraphico-musical lié à la collecte du miel (laquelle ne se fait que durant une saison qui n'était pas celle où je me trouvais là). Qu'il s'agisse des Pygmées de l'Itouri, de la Sangha ou de la Lobaye, tous sont grands amateurs de miel - sauvage bien entendu. Sa collecte s'apparente de près à une chasse, laquelle n'est menée que par deux ou trois individus, mais est préparée (ou plus exactement ouverte, semble-t-il) par un rituel chorégraphico- 


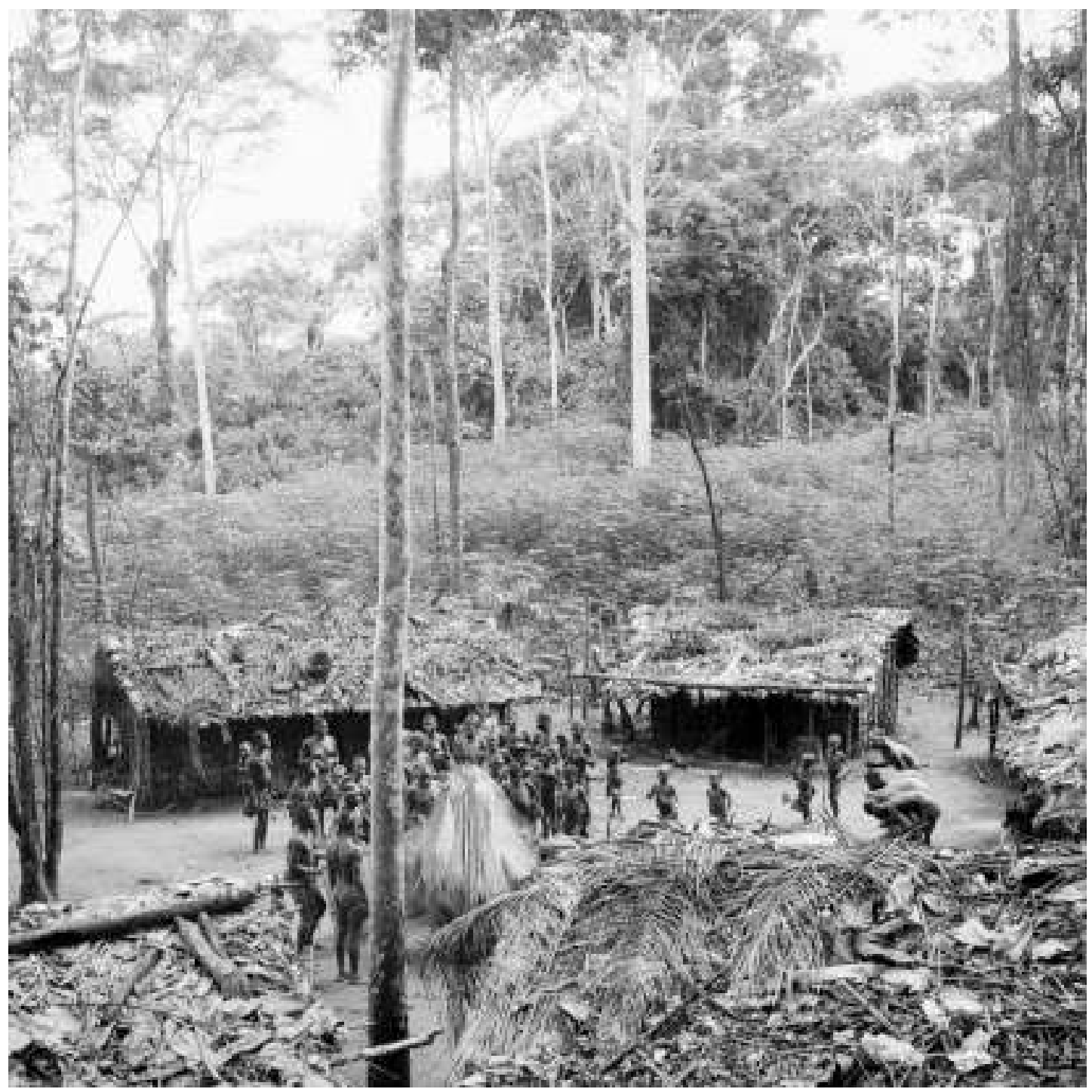

Cérémonie pour Edzingi, esprit de la forêt Vue générale du campement. Au centre, immobile, Edzingi, dressé de toute sa hauteur. À droite, penchés sur leurs instruments, les quatre tambourinaires.

25 septembre 1946. Le rituel a lieu non pas au sein de la grande forêt primaire qu'habitent en y nomadisant plus ou moins les BaNgombé, mais dans l'espace partiellement déboisé qu'occupe leur campement fixe, proche du village ngoundi de Gandicolo, campement reconnaissable à ses cases rectangulaires, totalement différentes des huttes hémisphériques en feuillage que construisent en forêt les Pygmées.

\section{TROIS RITUELS MUSiCAuX DES PygméEs BANgombÉ}




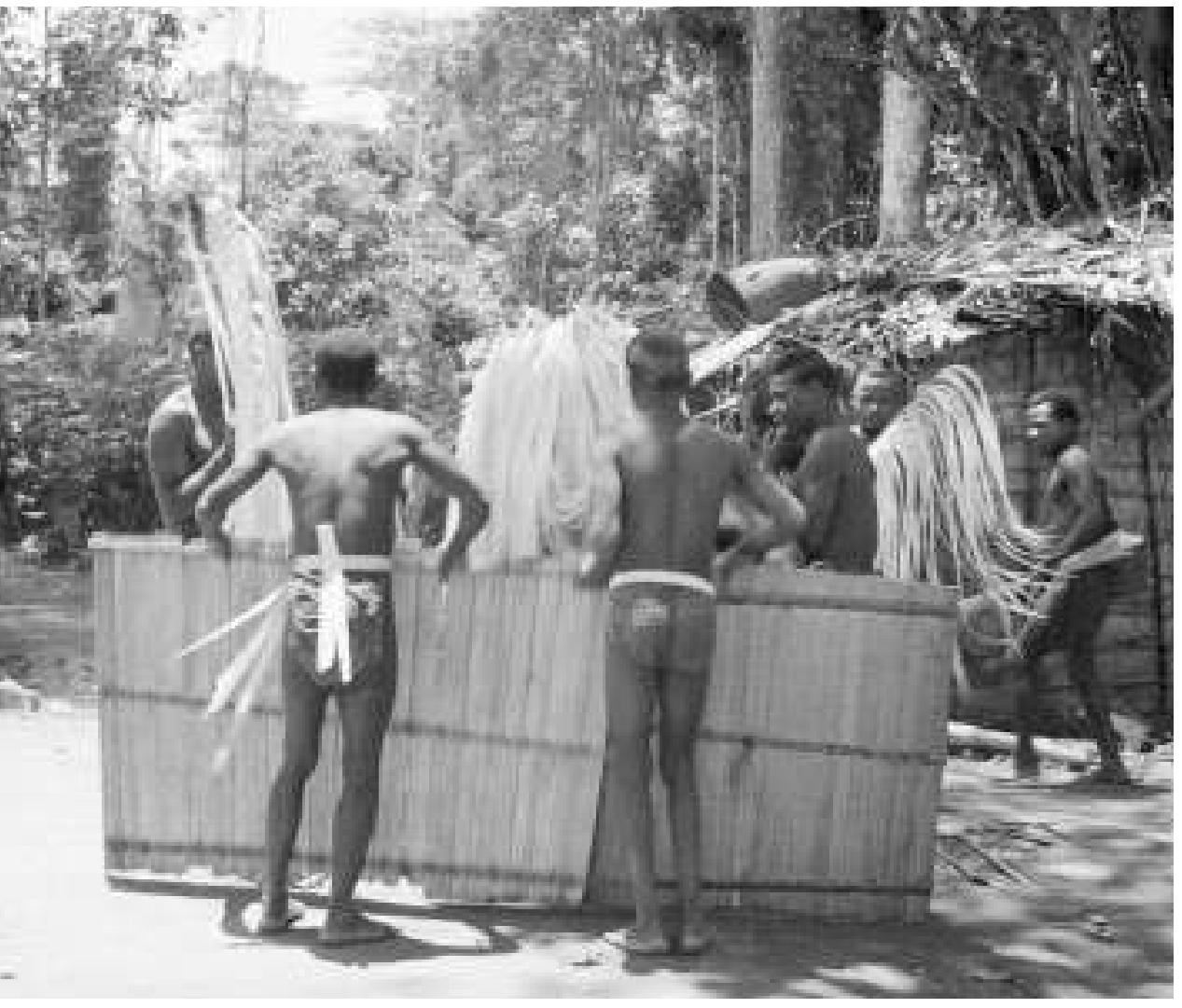

Dans ses

évolutions,

Edzingi est

constamment

escorté par

deux hommes

porteurs chacun

d'un écran léger,

visiblement

destiné à le

protéger.

Mais de quoi?

Du regard des

femmes qui

risqueraient

d'apercevoir

ses pieds?

II est ici

flanqué, à droite

et à gauche, de

deux hommes

agitant, l'un

et l'autre,

un grand rameau

de raphia frais.

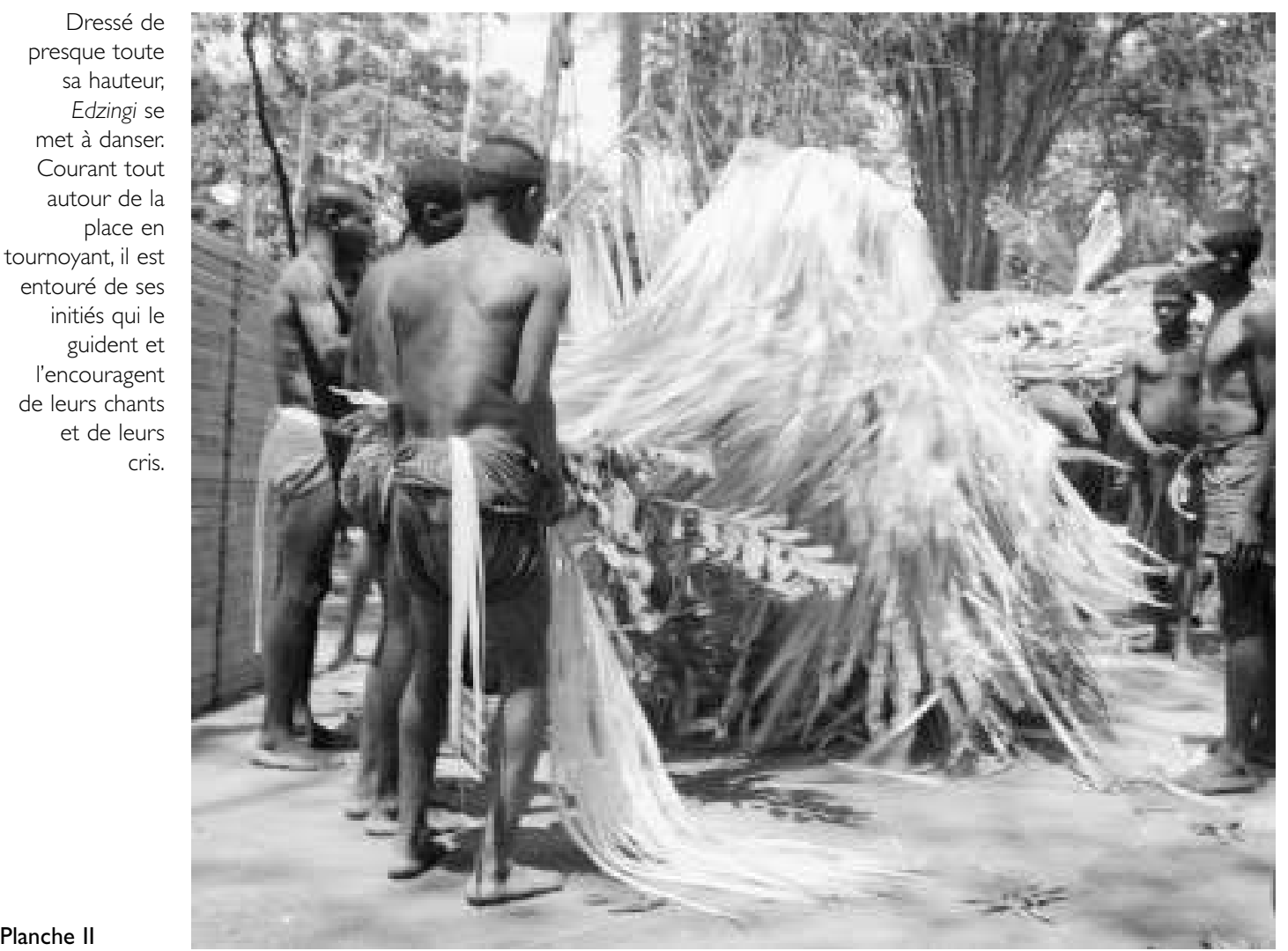



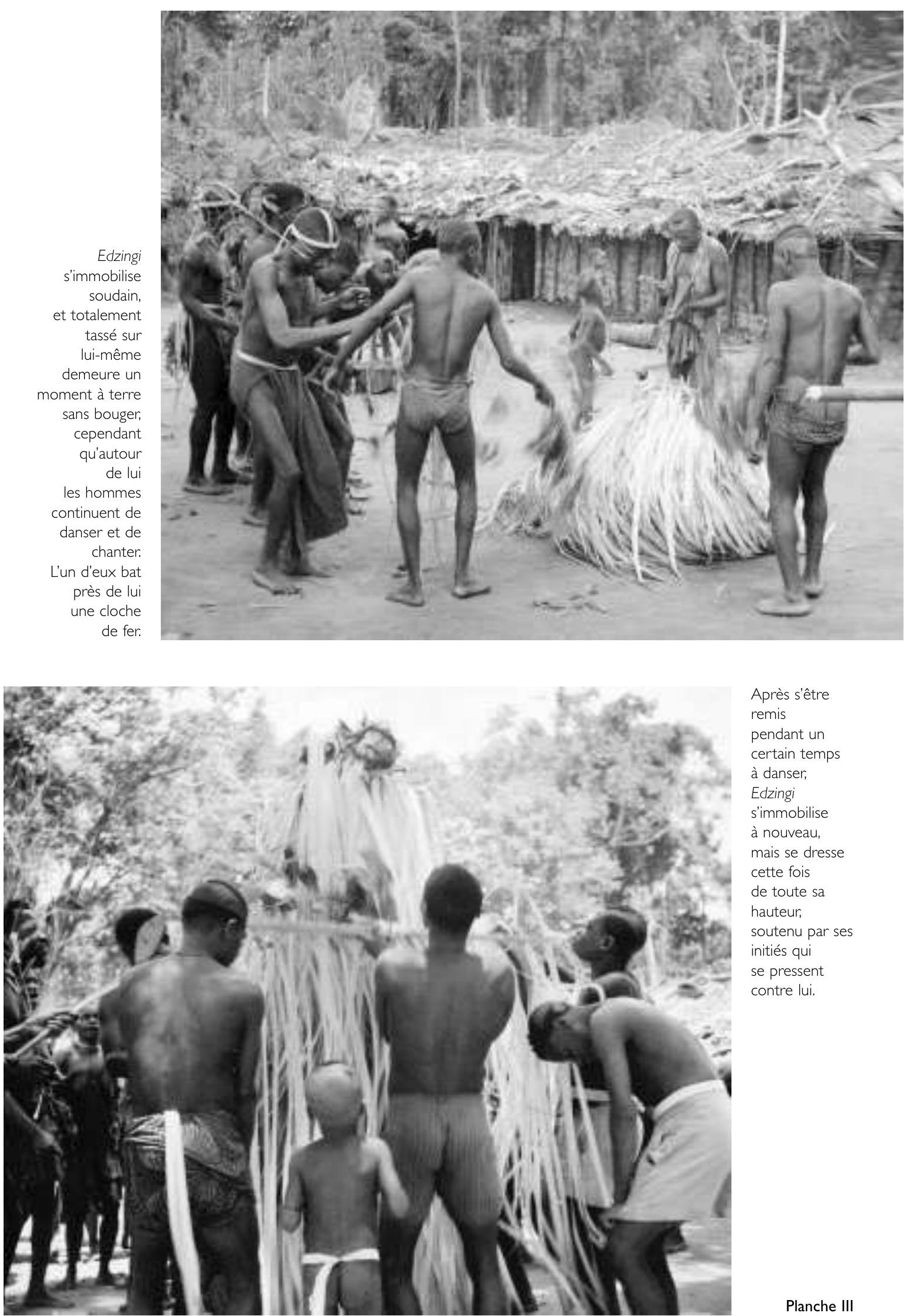

Après s'être remis

pendant un certain temps à danser, Edzingi s'immobilise à nouveau, mais se dresse cette fois de toute sa hauteur, soutenu par ses initiés qui se pressent contre lui. 

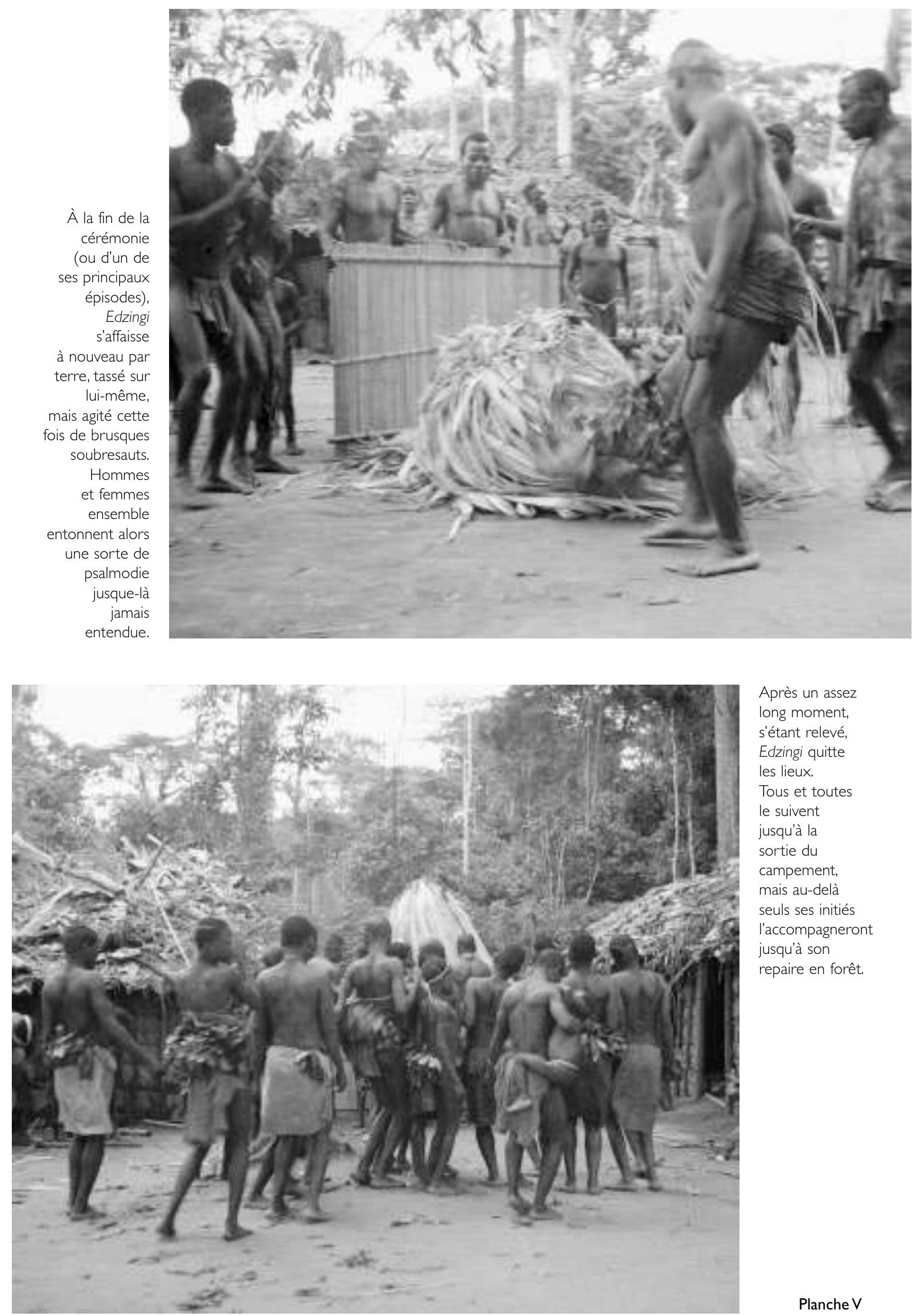

Après un assez long moment, s'étant relevé, Edzingi quitte les lieux.

Tous et toutes le suivent jusqu'à la sortie du campement, mais au-delà seuls ses initiés l'accompagneront jusqu'à son repaire en forêt. 


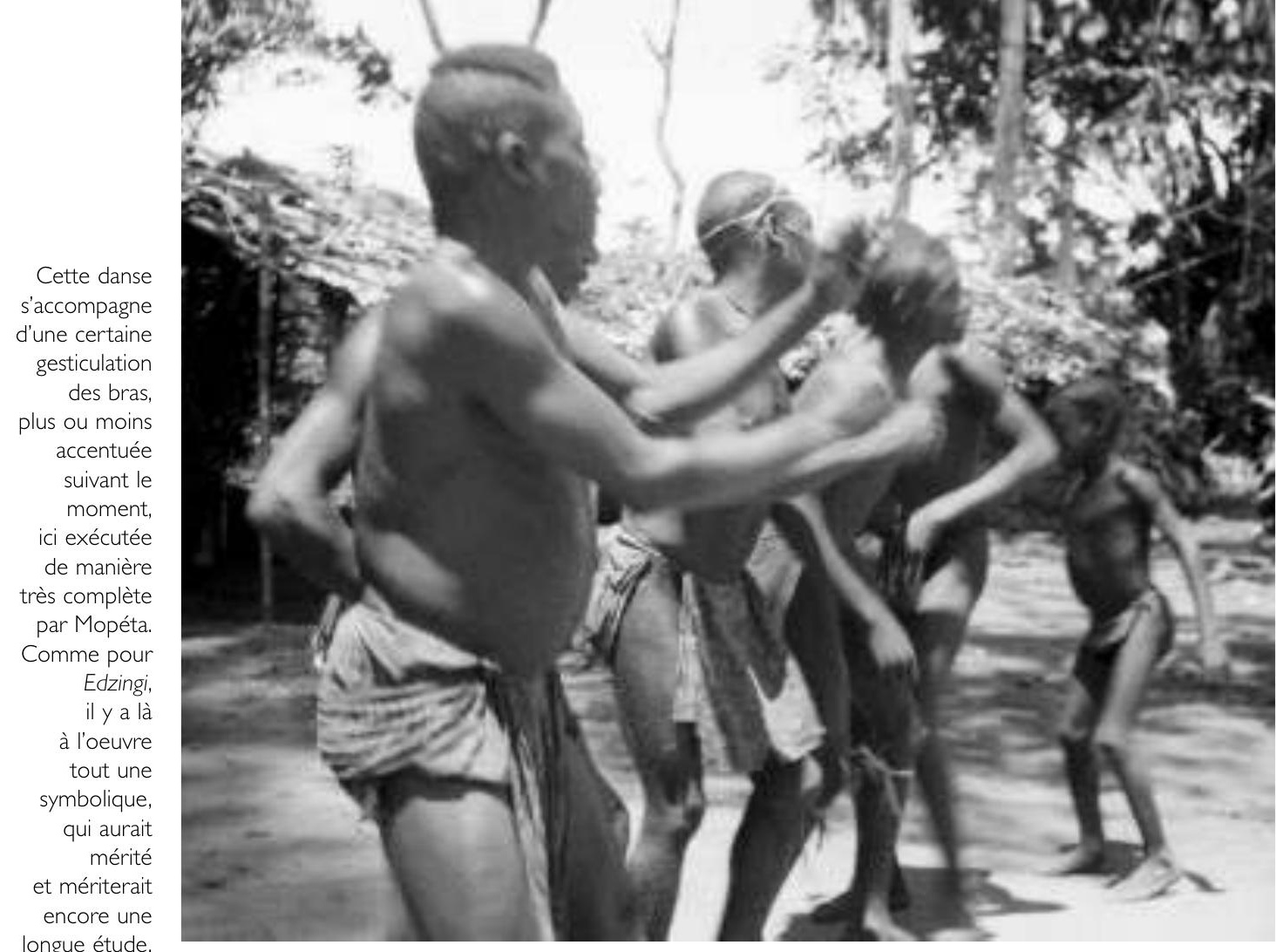

\section{des chasseurs}

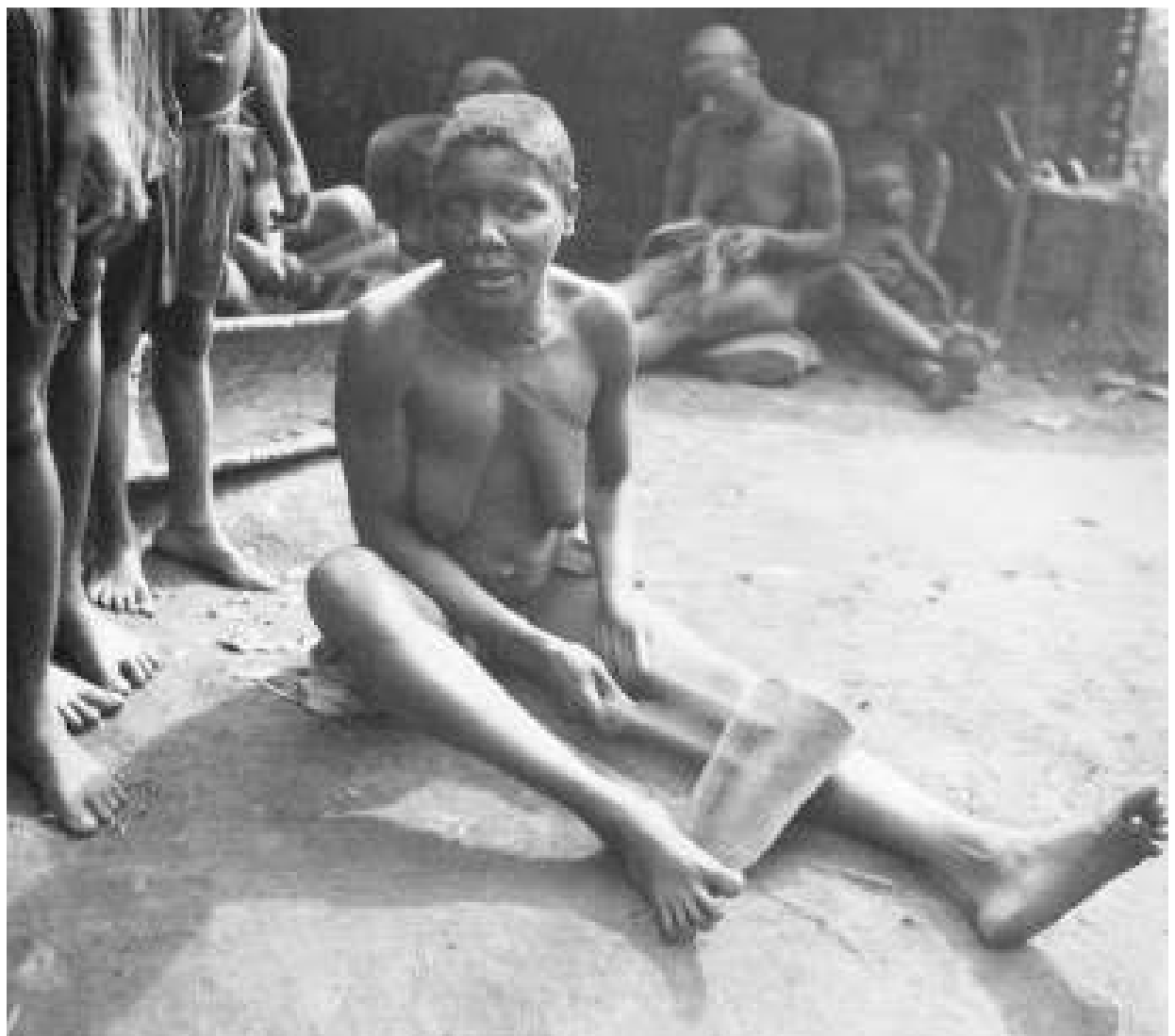

Dans le

groupe des

femmes, l'une

d'entre elles,

un peu à l'écart,

bat une cloche

de fer, plantée

dans le sol par le

manche et

maintenue en

place par un pied.

Derrière elle,

on aperçoit

l'ouverture

d'une auge

en bois, qu'une

autre femme

bat avec deux

baguettes. 


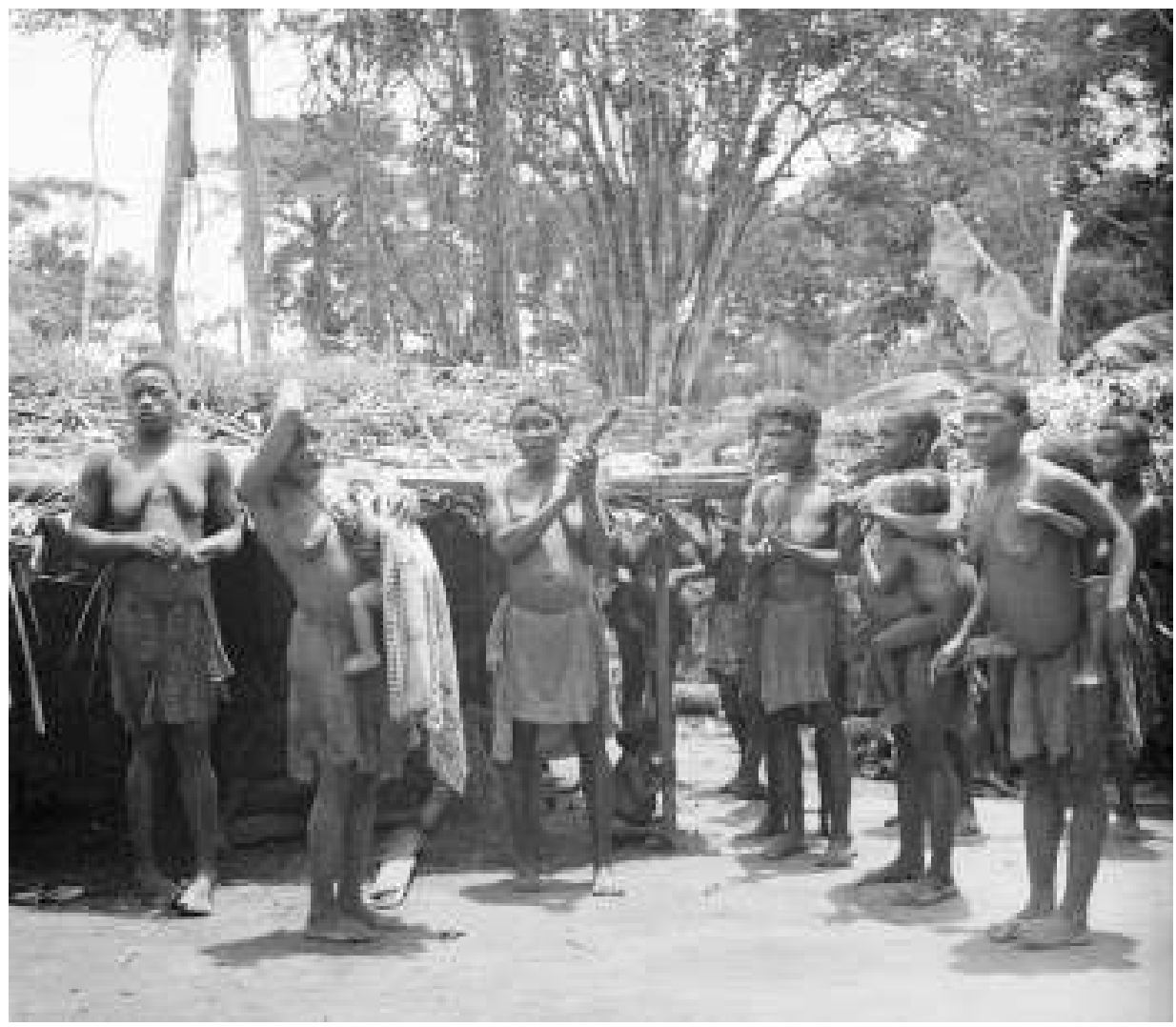

\section{Yeli, chant magique}

Femmes des chasseurs chantant en chœur yeli, chant magique qu'elles exécutent en vue du départ de leurs maris pour une chasse à l'éléphant. La soliste, bras replié, est la femme du chef.

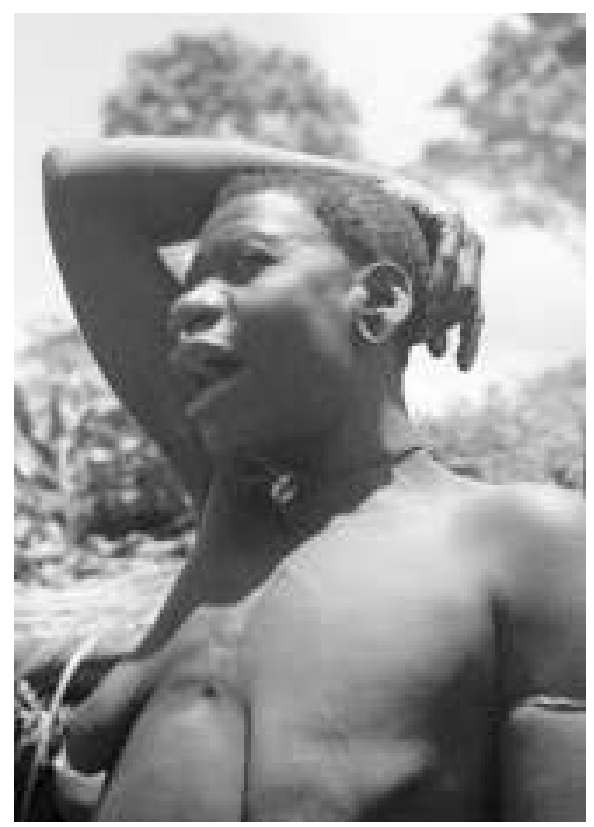

Autre femme chantant yeli, avec la position très particulière du bras replié sur la tête.

Corps tendu, nuque raide, attitude caractéristique de la danse de chasse dite bukela.

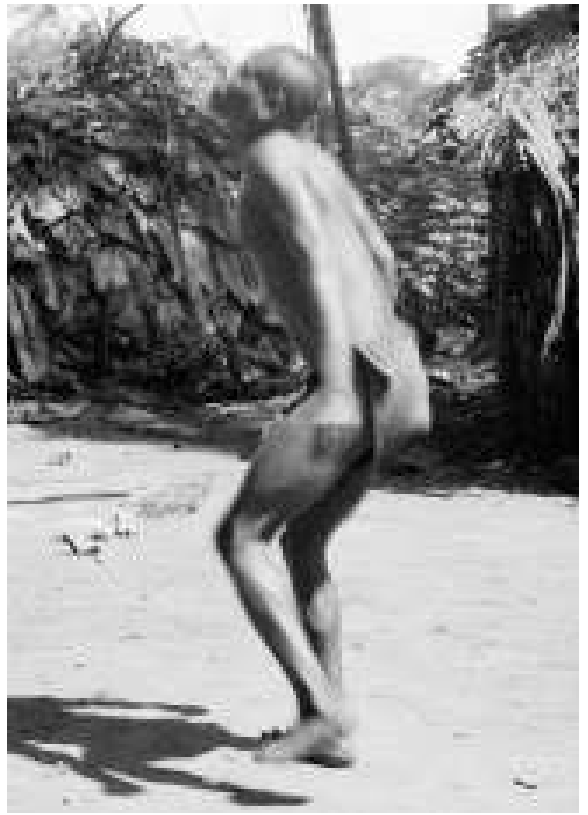


musical propitiatoire mobilisant le groupe tout entier. Observé chez des Pygmées Aka de la Lobaye - et non de la Sangha comme les BaNgombé, mais cela importe peu -, ce rituel a été décrit par Simha Arom (1978, disque 1B, plage $2 \mathrm{a}-\mathrm{k})$ et par Serge Bahuchet (1985: 214 sq.). De ces deux descriptions retenons ici d'une part que les divers chants, de styles très variés, qui les composent se font a capella (comme yeli, notons-le), d'autre part que certains d'entre eux sont des invocations aux mânes des ancêtres (Arom) et que d'autres accompagnent une action simulant un des temps forts ${ }^{16}$ de cette collecte (Bahuchet). D'un côté mânes, et donc culte, des ancêtres : religion; de l'autre simulation de l'entreprise projetée : magie, nous retrouvons là les deux dimensions constitutives, a-t-on vu, la première du rituel de Edzingi, la seconde de la danse de chasse bukela.

Cette magie en vue de la récolte du miel, les Pygmées de l'Itouri la mettent abondamment à l'œuvre sous diverses formes musico-chorégraphiques. Voici des extraits du long passage que Colin Turnbull (1963: 238-239) y consacre: «À toute heure, la forêt vibrait des chants et des danses qui symbolisent la Vie. Le jour, ils jouaient aux jeux réservés à la saison du miel [...]. Une fois le soleil couché ils dansaient la danse des abeilles [...]. Tandis que les hommes feignaient d'être à la recherche du miel et dansaient en une ligne sinueuse avec des gestes et une mimique exagérée [...], les femmes dansaient sur une autre file [...] prétendant être des abeilles. [...] Les deux files se rapprochaient graduellement l'une de l'autre; les femmes fredonnaient doucement en imitation du bourdonnement des abeilles ${ }^{17}[\ldots]$. Tous ensemble, ils entonnaient les chants magiques qui, s'élevant avec les volutes de la fumée, inciteraient les abeilles à venir leur apporter davantage de miel ».

Arrêtons-nous un instant sur les mots "chants magiques " qu'on vient de lire sous la plume de Colin Turnbull, tout à la fin de son ouvrage, car ils sont en pleine contradiction avec ce qu'est manifestement le fond de sa pensée. Or c'est elle qui nous importe. Parlant du molimo, qui est à la fois un " animal de la forêt " (1963 : 71) - personnage central de la religion des BaMbuti -, l'instrument de musique (paire des trompes droites) qui le caractérise et le cycle des cérémonies qui s'y rapportent, il écrit (ibid. : 70) : «Le molimo des Pygmées ne se rapporte [...] à aucune forme de magie ". Et, toujours dans le même chapitre, après avoir dit (ibid. : 80) que si la chasse est mauvaise, il faut, disent les Pygmées, réveiller la forêt - "Nous la réveillons par des chants, car nous voulons qu'elle se réveille heureuse "-, Colin Turnbull ajoute (ibid. : 81) : «Jamais leurs chants ne demandent telle ou telle chose, par exemple que la chasse soit fructueuse ou qu'un malade guérisse. Il suffit qu'ils réveillent la forêt et tout rentre dans l'ordre». Parlant, beaucoup plus loin (ibid. : 126), à propos de elima, leur autre grand cycle cérémoniel, "de la nuance subtile qui sépare la magie de la religion", il écrit : «Les BaMbuti ne croient pas du tout que c'est l'acte qui, d'une façon inexplicable, produit les résultats (ce que nous appelons magie), ils croient en un dieu bienveillant ou une puissance surnaturelle qu'ils identifient à la forêt ». Ce n'est donc pas, dans sa propre logique, "chants magiques" qu'il aurait dû dire à propos de la récolte du miel, mais « chants religieux ». Son lapsus linguae n'en est pas 
moins significatif. Retenons ici que, religion ou magie, Colin Turnbull nous met en présence d'une conception proprement pygmée de l'efficacité musicale. C'est là une donnée qu'il nous fallait impérativement prendre en compte.

Si sommaire qu'il soit, notre examen comparé de divers rituels menés par les Pygmées en vue du succès de la chasse aura permis de préciser le rôle que joue la musique. Elle y apparaît dans le cas de yeli comme le facteur principal, dans les autres cas comme l'auxiliaire des comportements religieux (culte des "mânes", des " esprits ", des " puissances surnaturelles») ou magiques (le semblable par le semblable) - en un mot des actions symboliques - qui les fondent. Prenons soin d'ajouter toutefois qu'auxiliaire de ces comportements, elle n'en est pas moins un composant indispensable et déterminant de l'action, car c'est elle qui donne sa forme au rituel et parce que sans forme celui-ci n'existerait tout simplement pas. "Actions symboliques musicales " donc, ces rituels de chasse sont à verser au dossier de ce que Claude Lévi-Strauss a si justement nommé l' "efficacité symbolique ", à propos d'un rituel de guérison amérindien, totalement différent de ceux qui nous occupent, mais musical lui aussi, car chanté de bout en bout.

Efficacité symbolique, disons-nous, mais aussi, bien sûr, pour les Pygmées et donc pour nous-mêmes, efficacité tout court. Or à quoi celle-ci tient-elle ? Ici, nos deux croyances, celle des Pygmées et la nôtre divergent. Les Pygmées croient, vient-on de voir, que le succès de la chasse dépend, soit du pouvoir des " esprits" de la forêt qu'ils invoquent (voie religieuse), soit des vertus de l'opération qu'ils mènent en simulant une chasse heureuse (voie magique), soit encore du mélange des deux. Ces croyances pygmées, nos convictions rationalistes nous empêchent malheureusement de les partager. En revanche, nous sommes tout prêts à croire, ce qui est différent, qu'elles affermissent les chasseurs dans leur conviction qu'ils vont trouver du gibier, qu'elles renforcent la confiance qu'ils ont en leur talent cynégétique et que par là même elles favorisent effectivement le succès de la chasse. Thèse totalement psychologisante, comme on voit, mais la psychologie ne fait-elle pas partie des conduites humaines? Telle sera en tout cas, provisoirement du moins, notre interprétation des choses.

Soulignons maintenant que, vues dans la perspective des Pygmées comme dans la nôtre, c'est à des représentations symboliques (aliquid stat pro aliquo) et à une efficacité du même nom que nous avons eu jusqu'ici à faire. Notre croyance est toutefois qu'en l'occurrence il n'y a pas que du symbolique en jeu. Mais avant d'y venir, il nous faut examiner un autre aspect des activités musicales des BaBinga.

\section{Une activité chorégraphico-musicale de simple divertissement}

Durant mon bref séjour auprès des BaBinga, en 1946, j'eus l'occasion d'assister à plusieurs danses qui n'étaient (en apparence tout au moins) que de simple divertissement ou de pure réjouissance. À côté de leur danse de chasse dite bukela, les BaNgombé en exécutaient une autre, dite $s a n d z 0^{18}$, en tous points comparable musicalement et chorégraphiquement, mais dont ils disaient euxmêmes qu'elle n'était pas pour «la viande», mais pour le plaisir. Pour ce qui est 
maintenant des BaMbenzélé, leurs voisins, djoboko ${ }^{19}$ était la danse exécutée en vue du succès de la chasse au filet, mais je la vis également dansée, pour le simple plaisir me fut-il dit, au cours d'une réjouissance célébrant la visite d'un campement venu des environs.

Brièvement décrite (à partir de notes très succintes, prises à l'époque, au cours de l'enregistrement), voici en quoi consistait la grande danse de divertissement, dite elanda ${ }^{20}$, que j'ai vu pratiquer par les BaMbenzélé, mais qui, selon toute probabilité, devait l'être aussi chez les BaNgombé. Le soir venu et les circonstances s'y prêtant, tout le monde s'était réuni pour danser sur la place laissée libre au centre du campement. Disposés sur deux lignes affrontées distantes de quelques pas, hommes et jeunes garçons d'un côté, femmes et jeunes filles de l'autre, danseurs et danseuses, chantant et battant des mains à intervalles réguliers, alternativement se rapprochaient de la ligne opposée ou s'en écartaient. La danse ne consistait guère qu’à se trémousser sur place, les hommes piétinant vigoureusement le sol, les femmes, et particulièrement les jeunes filles, effectuant de spectaculaires rotations du bassin, soulignées par les déplacements de la touffe de feuillage agrémentant leur postérieur. La musique pour elanda, purement vocale hormis les battements de mains, consiste en la succession de deux mouvements très fortement contrastés. Le premier est fait d'une brève formule mélodicorythmique répétée à satiété, chantée sans aucun jodel, polyphoniquement et à deux parties; celle des hommes à l'unisson et celle des femmes divisée. Le second est également à deux parties, cette fois totalement indépendantes l'une de l'autre. Celle des femmes et des jeunes filles constitue un véritable concert de cris, organisés en hoquet et mêlant des timbres très différents, aigus et suraigus. Celle des hommes consiste en une sorte de grondement rythmique court et formant un ostinato souligné par le bruit des pieds tambourinant en quelque sorte le sol. Constitutif du genre elanda, cet enchaînement de deux mouvements durait en moyenne entre cinq et huit minutes, le retour au premier mouvement chanté s'effectuant, après une courte période de flottement, en entonnant un autre air. $[N$. B. Un enregistrement de elanda (1946) figure dans le CD en fin de volume, \# 4.] Le spectacle que donne cette danse est celui d'un petit groupe s'amusant beaucoup et prenant plaisir à se dépenser physiquement. Exercice physique collectif auquel chacun concourt à sa manière, par la voix, par le souffle, par le geste, par le mouvement et l'engagement du corps tout entier. Exercice? Si incongru que cela paraisse, évoquons ici les essercizi de Scarlatti, eux aussi fondamentalement binaires et radicalement ludiques. Même esprit dans les deux cas, à ceci près qu'au lieu d'une seule personne, de dix doigts et d'un clavier, c'est un corps social d'un peu plus d'une vingtaine d'individus et de voix, d'une vingtaine de paires de bras et de jambes, qui est ici à l'œuvre. À ceci près également qu'ici la musique en tant qu'événement sonore n'est en fait pas le but principal de l'acteur multiple qu'on vient de décrire. Ces gens ne sont pas occupés à "faire de la musique». Passant alternativement par des moments de grande réussite et de complet cafouillage, celle-ci n'est pour eux qu'un moyen d'organiser l'action. Ce que le groupe a manifestement en vue, c'est le plaisir pris collectivement à la produire, 
bien plus que le produit lui-même. En bref, si inséparables qu'ils soient, c'est d'abord le musiquer qui lui importe, la musique comme résultat ne vient qu'après. Ce qui n'empêche pas qu'elle soit de temps à autre de très grande qualité et qu'alors elle soit très probablement, pour les musiciens - ou mieux les musiquants -, des moments d'une intense plénitude.

"Simple divertissement", ai-je dit de cette danse, car il ne m'en a rien été dit d'autre et parce qu'à l'inverse des trois actions musicales vues précédemment, explicitement données comme visant un objectif défini, il n'en a rien été de celleci. Cette danse n'en serait-elle pas moins, elle aussi, symbolique? La disposition des protagonistes en deux lignes affrontées, masculine et féminine, ne le donnerait-elle pas à penser? La question se pose. Mais ce serait en ce cas à leur insu, ce qui est bien différent de ce qui nous a jusqu’à présent occupé. Divertissement donc. Argument supplémentaire: Michelle Kisliuk (1998: 61) décrit, chez les Pygmées BaAka de la Lobaye, sous le même nom de elanda, une danse qui, autant que sa description très sommaire permette d'en juger, est la même que celle qui nous occupe ; l'écoute de la plage 9 de son CD II montre qu'il s'agit bien de la même musique. Or Michelle Kisliuk précise qu'il s'agit là d'une des rares danses (sinon la seule) «non censurée " par les Évangélistes qui sévissent dans la région, car elle « ressemble à un jeu et n’a apparemment pas de but spirituel ou efficace" (no apparent spiritual or efficacious purpose).

Divertissement, c'est le terme qu'emploie Pierre Sallée (dont on sait quel ethnographe rigoureux il était) pour désigner toute une suite chorégraphique et/ou musicale, composée de plusieurs mouvements, enregistrée chez les Pygmées Baka du Gabon (Sallée, discogr. 1975 et 1976). Là encore, il s’agit d’un jeu, compétitif certes, mais selon toute apparence purement ludique. Or deux de ces mouvements sont constitués par des pièces chantées a capella par des femmes et très proches du yeli des BaNgombé dont on a vu l'étroit rapport avec les rituels de chasse. Cela signifie que telle musique qu'on pourrait croire intrinsèquement chargée d'efficacité symbolique peut fort bien, dans d'autres circonstances, ne pas l'être. C'était, a-t-on dit, le cas de la danse (et de la musique) dite djoboko, exécutée par les BaMbenzélé de Gatongo en vue du succès de la chasse au filet, mais à l'occasion pur et simple support de la réjouissance. C'est aussi celui de la musique d'arc musical exécutée par des femmes. L'instrument est " utilisé essentiellement aujourd'hui lors des moments de divertissement" (Dehoux 1995: 80), mais «dans le passé », ajoute l'auteur, «son principal contexte d'utilisation était la chasse et plus précisément la chasse à la sagaie ». Par ailleurs, plusieurs pièces rangées par Simha Arom (1991 : 236) dans la catégorie « rituel » et présentées comme telles dans certains de ses disques, enregistrées dans d'autres circonstances, apparaissent ailleurs sous la dénomination de "divertissement ${ }^{21}$. Il n'y a pas à s'en étonner. Qu'une musique ou une danse liée à un rituel précis fasse couramment l'objet, avec ou sans restriction suivant les cas, d'un tout autre emploi - réjouissance, jeu, divertissement -, rien de plus banal et de plus universel.

Après avoir traité de l'efficacité symbolique de toute une partie du répertoire chorégraphico-musical des Pygmées, il importait de rappeler, comme on vient de 
le faire, que sa partie restante, dépourvue de tout souci exprès de finalité, occupe une place non moins importante dans leur vie quotidienne. On vient de voir que ces deux parties, rituelle et non rituelle, du répertoire se recouvrent très largement. Du point de vue de la pratique chorégraphico-musicale, qui va maintenant nous occuper, il n'y aura donc plus lieu de les distinguer et nous en traiterons désormais globalement.

\section{Pratiques: l'efficacité musicale socio-somatique}

Des différents points de vue à partir desquels, admet-on en général, il convient de considérer la musique ${ }^{22}$, deux seulement seront dans ce qui suit les nôtres. Notre propos étant ce qu'il est, au stade de la production qui est celui qui va nous occuper, deux choses sont en effet à considérer, dans leur dynamique commune bien entendu: d'une part l'événement sonore, produit d'une certaine action et vu dans ses très grandes lignes, de l'autre l'action productrice de l'événement, elle-même vue à travers les pratiques de ceux qui l'exécutent. En bref, d'un côté la musique et de l'autre, conjointement, le musiquer et les musiquants.

Commençons par rappeler, très brièvement car elles ont été abondamment décrites et sont bien connues, les principales caractéristiques de la musique pygmée. Celle-ci, essentiellement vocale, a pour première particularité son emploi systématique de la technique du jodel. Cela dit, elle est chorale, polyphonique et mixte (entendons par là que la plupart du temps les chœurs sont composés d'un mélange de voix d'hommes et de femmes). Trois instruments sont toutefois à mentionner, bien qu'inégalement répartis, d'Est en Ouest, le long de ce qu'on pourrait appeler le discontinuum pygmée : les sifflets, utilisés en hoquet avec la voix, l'arc musical, instrument féminin d'un type très particulier ${ }^{23}$, et la trompe à embouchure terminale, instrument masculin ${ }^{24}$, tous trois associés aux rituels de la chasse. Par ailleurs, notons que les Pygmées tambourinent, certes, mais que le tambour n'est chez eux, comme on sait, qu'un instrument d'emprunt, principalement employé dans leur rôle d'amuseurs publics. L'usage qu'ils en font n'a rien de remarquable. Les quelques autres instruments, tous d'accompagnement, dont ils se servent, ne leur sont pas propres et ne nécessitent pas qu'on en parle.

Venons-en maintenant au musiquer. Les Pygmées chantent et dansent beaucoup, avons-nous dit en guise d'introduction. Mais encore? Lors de mon séjour, en 1946, j'en étais venu à croire qu'hormis chasser et cueillir, manger, dormir et vaquer aux diverses occupations de la vie quotidienne, les BaBinga passaient d'une manière ou d'une autre le reste de leur temps à musiquer. Simple impression, bien sûr, mais que par la suite d'autres travaux, fondés sur une bien plus longue expérience que la mienne, devaient amplement confirmer. Tout d'abord, ceux de Colin Turnbull sur les BaMbuti de l'Itouri, bien connus en France grâce à la traduction (1963) de son The People of the Forest (1961). Organisé autour de deux grands rituels s'étendant chacun sur plusieurs mois, ce livre rend compte de manière extrêmement vivante de la place, de toute première importance, qu'y occupe la musique, et de son absolue quotidienneté. De son côté, en première 
page de sa célèbre Anthologie de la musique des Pygmées Aka (1978), Simha Arom note que chez eux, "il n'est pas de journée sans musique ». "La musique omniprésente», tel est par ailleurs le titre, hautement significatif, de la description générale qu'il a donnée plus tard de cette musique. Avec ses quatorze rubriques fonctionnelles, le tableau concluant cette description (1991 : 236) donne à voir l'ampleur et la variété de ce répertoire musical. Celui-ci fournit largement aux Pygmées, d'Est en Ouest de la grande forêt équatoriale, de quoi musiquer chaque jour et en toutes circonstances, rituelles ou non.

Avant d'en venir à leur comportement de musiquants, rappelons succinctement quel genre d'existence ils mènent et quel type de comportement quotidien il leur impose. On sait qu'ils vivent par petits groupes (une trentaine de personnes ${ }^{25}$, quarante tout au plus) isolés en forêt et formant autant de petites communautés très compactes, éloignées les unes des autres, quasiment acéphales, et où les impératifs de la vie pratique (chasse et collecte, semi-nomadisme), combinés à ceux de la tradition, assignent à chacun, homme ou femme, son rôle, mais en le laissant, dans ce cadre, très largement maître de ses initiatives. Au sein du groupe, le fil des jours ne va bien sûr pas sans conflits, mais le système fonctionne, c'est du moins la réputation qui lui est faite, de manière à toujours les régler pacifiquement. Isolement très réduit de l'individu, vie communautaire intense, menée dans une grande intimité physique des corps, au demeurant très peu vêtus - promiscuité, serait-on tenté de dire, si le mot n'était si dépréciatif -, solidarité, complémentarité, respect mutuel, responsabilité de chacun à l'égard des autres, telles sont les modalités de l'existence quotidienne.

Dans semblable contexte, que ce soit naturel ou culturel, on ne chante pas tout seul : on chante soit en chœur si l'on est ensemble, soit en dialoguant si l'on est éloigné les uns des autres (en partant à la chasse au filet notamment ${ }^{26}$ ), mais soulignons-le, car cette particularité est essentielle, jamais à l'unisson ${ }^{27}$. Il en résulte une polyphonie ${ }^{28}$ à deux, trois ou quatre parties suivant les circonstances, chaque partie étant naturellement plus ou moins redoublée suivant que les participants sont plus ou moins nombreux. Dans la conduite de ces diverses parties chantées, les Pygmées font preuve de cette indépendance dans l'interdépendance dont on vient de dire qu'elle caractérisait leur manière de se comporter dans le quotidien de la vie pratique. Il y a là une homologie des deux ordres de comportement, musical et social, extrêmement significative et qui a été parfaitement décrite par Simha Arom (1991 : 229-231).

Ce que l'on souhaite souligner ici, c'est que chanter de cette manière, mener sa partie à la fois en la distinguant de celles des autres et en la règlant sur elles, le faire de plus avec une certaine part d'improvisation, donc d'imprévu, requiert naturellement de chacun qu'il soit à l'écoute à la fois de lui-même et des autres et qu'ainsi son engagement corporel, générateur de cet état vibratoire qui est par définition celui de la personne qui chante, soit à la fois constant et total. Engagement qui semble en même temps aller de pair - ce n'est pas contradictoire - avec un certain oubli de soi-même, tant l'individu chantant paraît faire corps avec le reste des chanteurs. Tout se passe donc comme si le groupe se 
muait en une seule grande soufflerie, faite d'une multiplicité de bouches ne constituant plus qu'un seul tout organique, régi nonobstant sa complexité par un seul et même système de résonance.

Mais, dira-t-on, n'est-ce pas là le propre de toute bonne chorale? Qu'y a-t-il là de particulier aux Pygmées? Il y a, tout d'abord, le fait pour cette pratique d'être à la fois si quotidienne et si souvent intégralement collective, mobilisant comme elle le fait très fréquemment la totalité du campement, hommes et femmes, jeunes et vieux réunis. Ensuite le fait, d'une part de laisser à chacun, à l'intérieur d'une action commune à la fois contraignante et diversifiée, une grande marge de liberté individuelle, d'autre part d'enchaîner ad libitum à la partie chantée une partie criée, et d'alterner ainsi polyphonie et hoquet. Enfin, le fait de recourir de manière très variée au jodel, technique en rapport, avons-nous vu, avec l'acoustique de l'environnement, mais caractérisée surtout, au niveau de la production, qui est celui qui nous importe, par ses particularités articulatoires. Celles-ci résultent de la mise en œuvre alternée de deux types d'émission vocale, voix de tête et voix de poitrine, et par là-même d'un engagement corporel remarquablement étendu. Par ailleurs, si la musique, principalement vocale, doit être vue comme étant par excellence - reprenons l'heureuse formule de Marcel Mauss - une "technique du corps ", musiquer à la manière des Pygmées nous met en présence d'une véritable "technique du corps social ", puisqu'en l'occurrence c'est bien le corps social tout entier qui est concerné. En conséquence, si chanter est par nature une activité "somatique» - je veux dire par là pas seulement corporelle : le corps, mais en même temps la vie qui l'anime -, et plus précisément une "mise en résonance du corps", comme l'écrit Bernard Lortat-Jacob (Bouet et al. 2002: 215), alors il convient de considérer que musiquer comme le font les Pygmées constitue (qu'on me passe la barbarie du terme, il dit bien ce qu'il veut dire) une véritable activité "socio-somatique», mettant «en résonance» le corps social tout entier. Avant d'aller plus loin, revenons un instant sur le terme "somatique», pour préciser que son emploi ne vise en aucun cas à réduire le chant et la danse des Pygmées à des activités purement et simplement physiologiques. Musicales, celles-ci mettent en œuvre, par définition, des fonctions du plus haut niveau, sensible et intellectuel. Leur dimension concrète, faite d'individus de chair et de sang, n'en est pas moins essentielle. Or c'est elle qui présentement nous occupe.

Musiquer, a-t-on vu, consiste fréquemment pour les Pygmées, en même temps qu'à chanter, à danser. Là encore l'indépendance de chacun va de pair avec l'unité de l'ensemble. Le concert des mouvements est comme celui des voix : chaque individu y concourt en en respectant la structure générale et en tenant, avec une certaine marge de liberté, le rôle que lui attribuent l'âge, le sexe ou la fonction. Les différents membres composant le corps social participent ainsi, chacun suivant son statut, au chant et à la danse. Corps individuel, corps social, suivant que l'un ou l'autre est concerné, c'est dans les deux cas du comportement d'un être vivant, engagé dans sa totalité corporelle, qu'il s'agit ; c'est donc bien de somatique et de socio-somatique qu'il convient de parler. Mais dira-t-on ici encore, n'est-ce pas ce qui se passe dans toute danse chantée, celle des Bretons, pour ne 
citer que cet exemple? L'activité chorégraphico-musicale des Pygmées présente en fait un faisceau de particularités qui n'appartiennent, semble-t-il, qu'à elle et qui consistent, résumons : 1) à mobiliser la plupart du temps la société tout entière ; 2) à toujours diviser les rôles, tant chantés que dansés ; 3) à prendre place quasi quotidiennement ; 4) à avoir en vue aussi bien un pur et simple divertissement qu'un résultat vital et précis, comme le succès de la chasse ou la bienveillance des "esprits ». Cela étant, on est en droit de considérer que musiquer occupe dans la vie de cette population une place d'une importance et d'une nature tout à fait exceptionnelles, ce qui mérite réflexion.

En effet, il y a manifestement quelque chose de paradoxal à ce qu'au dénuement matériel dans lequel vivent les Pygmées, à ce qu'à la précarité29 de leur quotidien, corresponde une telle abondance d'activités musicales. La disproportion a de quoi surprendre. On s'attendrait à ce que la pression des tâches journalières laisse peu de place et peu de moyens pour des actions sans utilité immédiate apparente. Et si celles-ci, nous demanderons-nous, en avaient bien au contraire une, autre que cette efficacité symbolique et que ce pur et simple plaisir dont nous avons jusqu'ici parlé ?

Pratiquement, que se passe-t-il ? Il est clair que chanter et danser autant et de cette manière ne peut manquer d'avoir des effets, physiologiques et psychologiques - en un mot somatiques -, tant sur l'individu pris isolément que sur tous ceux qui composent le groupe, et par là sur le groupe lui-même, pris dans son ensemble. Musique: fait de culture. Mais musiquer au point où le font les Pygmées apparaît tout autant, et dans le sens fort du terme, comme un fait de culture physique : exercice quotidien de mise et de maintien en forme du corps, tant individuel que social. Aux deux niveaux, le résultat de cet exercice intensif de la musique - de ce musiquer - a nécessairement pour résultat, qu’il soit ou non consciemment recherché, d'entretenir la santé physique et morale au sein de cette micro-société qu'est un campement pygmée.

Résultat consciemment ou non recherché, vient-on de lire. Mais l'un ou l'autre, n'est-il pas important de le savoir? Pour répondre à la question, procédons brièvement par analogie. "Parler», chacun de nous, lorsqu'il parle conviendra sans difficulté, Pygmée ou non, que c'est ce qu'il est en train de faire, sans être pour autant conscient, bien que ce soit le cas, que ce faisant il est en train de «communiquer». Toutes choses égales, la situation qui nous occupe est la même. Chanter et danser, chacun dira sans hésiter, s'il le fait, que c'est ce qu'il fait. En revanche, le même chacun peut parfaitement ignorer, ou en tout cas ne pas dire, que ce faisant il est en train de cultiver sa forme physique, en même temps que celle de la collectivité. Or dans le cas du Pygmée c’est précisément et manifestement, qu'il le dise ou non, le résultat de ce qu'il est en train de faire. Tout se passe donc comme si cette recherche de la santé collective était bel et bien l'objectif conscient ou non, peu importe -, de cet usage si continu et si systématique de la musique, ou plus exactement du «musiquer », par les Pygmées. C'est pour mettre en évidence cet aspect fondamental de leurs pratiques chorégraphico-musicales qu'il a paru nécessaire de faire appel à la notion d'efficacité musicale somatique. 


\section{Musiquer : une activité non de surplus mais de survie}

Dans son livre, Les Pygmées Aka et la forêt centrafricaine, Serge Bahuchet indique (1985: 530) que le verbe signifiant " être heureux » a pour premier sens "donner la réponse (dans le chant) ». Indication précieuse qui montre que l'idée de bonheur est étroitement liée, dans l'esprit de ces Pygmées, à leur pratique du "chant polyphonique».

"Être heureux", ajoute-t-il, "c'est être d'accord, être en harmonie avec les autres ». Se fondant essentiellement sur ces deux notions, chanter polyphoniquement et être heureux ${ }^{30}$, il écrit, en conclusion de son article intitulé «De la musique considérée comme une philosophie (chez les Pygmées Aka de Centrafrique)", paru dans le volume d'hommage à Simha Arom aux travaux duquel il se réfere abondamment, ce qui suit: "Pour les Pygmées Aka, le chant polyphonique est donc un reflet de la communauté en même temps qu'une communion, d'essence religieuse. Placée au cœur de la vie sociale, la musique est fondée sur les valeurs fondamentales de cette société forestière; en ce sens, la musique est véritablement une philosophie. " Sans manquer au respect dû à Serge Bahuchet et à sa grande connaissance des Pygmées, j’aurais pour ma part tendance à interpréter différemment les choses. Du chant polyphonique je préférerais dire qu'il constitue, plutôt que le « reflet de la communauté », le " ressort de la vie communautaire». Et au lieu de voir dans l'usage que les Pygmées font de la musique une "philosophie", autrement dit une construction intellectuelle résultant d'un ensemble de choix conscients et organisés, j’y verrais, ce qui est bien différent, une technique. Mousikê technê, "technique musique" (i. e. "des Muses ») auraient dit les Grecs. Bref, dans le sens fort du terme, une praxis. Une réponse certes culturellement très élaborée, mais également instinctive - l'un n'empêche pas l'autre - à une situation donnée : celle de la vie que les Pygmées mènent en forêt et que celle-ci leur impose.

Que chasser et musiquer ne soient pour eux que les deux faces de leur combat pour survivre, s'ajoutant à ce qui en a été dit jusqu'ici, un fait rapporté par Colin Turnbull l'illustrera particulièrement bien. Les BaMbuti de l'Itouri consacrent, at-on vu, pendant des semaines de longues cérémonies à un esprit de la forêt nommé molimo. Durant tout ce temps, «les hommes doivent d'abord chanter, car, selon leurs paroles, le "véritable travail" du molimo, c'est cela, manger et chanter, chanter et manger ", écrit Colin Turnbull (1963: 70). Or une des opérations quotidiennes du rituel consiste à quêter de la nourriture et à en remplir un panier destiné aux participants. On lui " expliqua ", note-t-il (ibid. :71), que «manger était [pour les hommes] un devoir, de même que c’était un devoir pour un adulte mâle de ne pas s'endormir et de continuer à chanter tant que les chants du molimo [qui ne doivent pas cesser de la nuit] étaient en cours». Obligation si stricte, précise-t-il, que «l'un des plus grands crimes que puisse commettre un Pygmée mâle - sinon le plus grand - est de s'endormir durant les chants du molimo». On ne peut dire plus clairement que pour les BaMbuti chanter et manger sont à égalité ce qui permet de rester en vie, autrement dit de survivre. 
Mais de nos jours, pour les Pygmées, le combat dont on vient de parler revêt un nouvel aspect. Comme pour toutes les « ethnies minoritaires » dont ils font partie - et dont Survival International, précisément, se voue à défendre les droits -, il porte principalement, désormais, sur une tout autre dimension de la survie. Celle de la survie, disons sommairement, identitaire. La vie des Pygmées était si étroitement liée aux conditions matérielles de leur existence - grande forêt, chasse, cueillette - que celles-ci disparaissant, leur identité comme peuple se définissant par lui-même est à présent tragiquement menacée. Quittons par conséquent le "présent intemporel » dont on a parlé au tout début de ce texte et dans lequel nous avons jusqu'ici évolué, pour en venir à celui d'aujourd'hui. D'un aujourd'hui qui est celui d'un drame parfois cauchemardesque ${ }^{31}$. On nous pardonnera, espérons-nous, d'en donner dans ce qui suit une image à tout prendre aimable et touchante.

On sait que les Pygmées vivent depuis fort longtemps, avec leurs voisins "Grands Noirs " (terme consacré), dans des relations de vassalité. Or celle-ci tourne de plus en plus, dans le présent contexte, à l'asservissement pur et simple. Ne songeant qu'à les faire travailler davantage à leur profit, leurs "maitres ", nous apprend Michelle Kisliuk (1998 : 149), font pression sur eux pour qu'ils renoncent à chanter et à danser "les jours de semaine ". "Danser en semaine! " s'indignent-ils. Comment, si tristement réduits à l'état de prolétaires, ces Pygmées peuvent-ils encore chanter et danser ? Nous demanderons-nous. Insouciance? Pur attrait du plaisir? Bien sûr! Mais, faut-il penser, cette conduite tient sans doute bien plus à ce que, les ravages de la déforestation ayant mis fin à leur mode de vie traditionnelle, musiquer, activité si intimement liée à la chasse et si étroitement fondatrice de la vie communautaire, constitue pour eux, symboliquement et concrètement, le seul moyen de demeurer eux-mêmes. Autrement dit, demeure leur principal espace de survie ${ }^{32}$.

"La musique, de tous les passe-temps le plus beau ", disait, à la Renaissance, le poète ${ }^{33}$. "Musiquer, de toutes les activités la plus indispensable ", disent à leur manière, de nos jours encore, les Pygmées. Surplus dans le premier cas. Survie dans le second. Deux définitions opposées, mais également significatives de la musique et par là même de la société concernée. Resterait à dire pourquoi la musique des Pygmées est, ou nous paraît, si belle.

Musiquer? Pour les Pygmées il s'agit là d'une véritable technique de l'existence et de la survie collective. Telle est la thèse. Il est clair qu'elle est, en fait, invérifiable. Elle n'en a pas moins pour elle - les données invoquées étant présumées exactes - d'être, croirais-je, à la fois plausible ${ }^{34}$ et économique ${ }^{35}$.

MOTS CLÉS/KEYWORDS : Afrique/Africa - Pygmées/Pygmies - pratiques musicales/musicking symbolisme/symbolism - efficacité somatique/somatic efficiency. 
1. «Pygmées": on me pardonnera l'emploi de cette appellation, souvent critiquée, aux ÉtatsUnis surtout, parce qu'entachée parfois d'une certaine connotation méprisante. Il est à peine besoin de préciser qu'elle en est ici totalement dépourvue. Pour avoir moi-même fréquenté, si brièvement que ce soit, des Pygmées et pour avoir depuis beaucoup lu à leur sujet - l'un confirmant l'autre -, je les tiens au contraire pour des exemples d'humanité tout particulièrement respectables. Cela dit, l'usage fait ici de cette appellation présente, en regard de l'inconvénient qu'on vient de dire, trop de commodité pour qu'on y renonce. Par ailleurs pourquoi, parlant des Pygmées en général, n'utiliser, comme on va le faire, que le pronom "ils " ? Les femmes seraient-elles exclues de la problématique? Que le lecteur ne me tienne pas pour machiste et politiquement incorrect : l'usage en français veut que, sauf si la spécification "elles" s'impose, le pronom masculin pluriel vaille pour les deux genres.

2. La forêt primaire, leur habitat naturel, est dévastée. La chasse à l'éléphant, leur grande fierté, leur est interdite. Pour ce qui est des BaAka de la Lobaye, constatant que "leur milieu naturel » disparaît, Serge Bahuchet écrivait en 1985 (p. 579) : "Ils n'ont plus de place, ni dans ce milieu, ni dans ce monde. Ils disparaîtront à leur tour". Vingt ans plus tard, en 1998, Michelle Kisliuk leur promet au contraire (p. X) « un futur florissant et à voies multiples ", mais à condition [rien de plus simple, en effet!] qu'ils "redéfinissent leurs propres identités». On appréciera. Cf. infra, note 31 .

3. "Esprits» de la forêt: principalement Edzingi chez les Pygmées de l'Ouest (cf. note 6), Molimo chez ceux de l'Est (cf. note 24).

4. Sur la Mission Ogooué-Congo, voir Noël Ballif (1954), qui en a été l'organisateur. Notre séjour chez ces Pygmées (mais plus souvent dans les deux villages de "Grands Noirs", Ngoundi et Pomo, dont ils dépendaient, que dans leurs campements sis à proximité) a été très bref : six semaines, du 26 août au 6 octobre 1946. Gandicolo, village ngoundi installé au bord de la Sangha, et Gatongo, village pomo, proches l'un de l'autre, sont situés un peu au nord de Ouesso, en République du Congo, à l'époque Moyen-Congo. ments de la musique des danses dites djoboko, elanda et bandjo (33 faces de disque); sur les BaNgombé (dépendant des Ngoundi de Gandicolo): danses dites nabula, bukela, sandzo, rituel pour Edzingi, chœur dit yeli (50 faces de disque), auxquels s'ajoutent 33 faces d'enregistrements divers : cris de chasse, chants hors situation, documents linguistiques. C'est de chez ces BaNgombé que proviennent en grande partie les enregistrements recueillis par Herbert Pepper autour, croirais-je, de 1952, et publiés aux alentours de 1958, dans sa grande et historique Anthologie de la vie africaine. Ces BaNgombé, récemment établis à proximité de Gandicolo, venaient, rive droite de la Sangha, du Cameroun.

6. Edzingi (Sallée 1975: face B-3, Tsinghi; Bahuchet 1985: 78-79, 435, 455, 457, Zèngi; Kisliuk 1998, Edjengi, Njengi; Kubik 2004, Jenge). L'enregistrement d'une séquence musicale pour ce rituel figure dans l'Anthologie de la musique des Pygmées Aka (disque 2 B-1) de Simha Arom, mais y est donné sous le nom de mokondi, nom générique des entités religieuses de ces Pygmées. La cérémonie concernée a trait à "l'établissement d'un nouveau campement ", circonstance pour laquelle il est fait appel à ce génie de la forêt, "Esprit supérieur, maître des mânes" selon Bahuchet (ibid. : 435). Pour sa part, Michelle Kisliuk, qui rapporte d'intéressantes données sur les récents avatars de ce rituel, en présente deux enregistrements (CD 214 \& 19). De leurs côtés, Daisaku Tsuru (1998) et Daisuke Bundo (2001) font l'un et l'autre abondamment état de jengi, qu'ils donnent pour être, de loin, la plus importante "spirit performance" des Baka du Cameroun.

Signalons que la musique pour le rituel de Edzingi, très brièvement décrit ici, a fait l'objet du plus long enregistrement réalisé au cours de la mission Ogooué-Congo : suite de 24 faces de disques, soit, en gros, une heure quarante. Douze épisodes en ont été publiés par mes soins sous étiquette du Musée de l'Homme $(78 \mathrm{tm} / 30 \mathrm{~cm})$. Une face entière du disque microsillon Musique Pygmée de la Haute-Sangha (33tm/17cm), y est consacrée. Par ailleurs, le film Danses congolaises, tourné à cette occasion par les cinéastes de la mission, comporte une (trop) brève séquence donnant à voir la beauté de l'exceptionnel spectacle chorégraphico-musical que compose Edzingi. Ajoutons qu'à ma connaissance, à l'exception d'un texte, tout récent, de Kubik (2004 : 100 sq.), aucun des tra- 
vaux ultérieurs sur la musique des Pygmées n’a fait mention d'une quelconque de ces publications, dont l'ensemble n'en constitue pas moins la plus ancienne documentation (1946) relative à un rituel qui occupe une place centrale dans l'univers symbolique des Pygmées. Il n'était donc pas inutile d'en signaler l'existence.

La présente cérémonie de $E d z i n g i$, qui s'étendit sur deux jours, les 25 et 26 septembre 1946, a été faite à ma demande ("sur commande» donc), moyennant divers cadeaux négociés, par l'intermédiaire à la fois de mon interprète et du chef de Gandicolo, tous deux "propriétaires » de Pygmées, avec Wamata, doyen du campement voisin des BaNgombé. Noël Ballif (1954 : 204205) en a fait un bref récit, illustré (page 208) d'une très belle et très représentative photo.

7. Bukela a été enregistré au cours d'une danse de routine, spontanément exécutée par les BaNgombé à la suite d'une autre danse dénommée sandzo, sur laquelle on reviendra plus loin, cf. note 18 .

8. On m’avait beaucoup parlé, à Gandicolo, du yeli des femmes bangombé. Je me réfere ici à un yeli qui a été exécuté à ma demande, en vue de son enregistrement. Michelle Kisliuk (1998: 68), parlant de Djoboko mentionne un « rite de femmes appelé Yeli, visant à la bénédiction et à l'abondance du gibier». Daisaku Tsuru (1998: 65) y fait une brève allusion. Sur le mot yeli, voir plus loin note 14. Divers yeli de 1946 ont été publiés: Ethnic Folkways 1947, Musée de l'Homme 1948, Boîte à Musique 1948 et 1957, Columbia Lomax 1951, Peabody Museum et Musée de l'Homme 1957. Deux transcriptions en ont été proposées, l'une par Yvette Grimaud (1957: 12 \& IV), l'autre (de Folkways 1947) par Rose Brandel (1961 : 182-183).

9. Comme on sait, le jodel est un des traits les plus caractéristiques du chant pygmée. Dans son article "À propos du jodel ", Constantin Brailoiu (1949: 71), qualifiant de "trouvaille capitale" le jodel rapporté par la mission Ogooué-Congo, l'intègre à la problématique générale du jodel formulée par Hornbostel et montre qu'en conséquence une révision de celle-ci s'impose.

10. Abstinence sexuelle signalée par Paul Schebesta (1940 : 35-37) à propos de la chasse à l'éléphant.

11. "Avant leur départ» selon certains, «durant toute leur absence» selon d'autres.
12. Interprète nommé Ambenga, Ngoundi du village de Gandicolo et "propriétaire ", comme il le disait lui-même, d'un BaNgombé.

13. Lucien Demesse (1980: 5), qui dans les années 1950 a longuement travaillé chez les Pygmées de la Sangha, projetait de consacrer «le troisième volet " de son œuvre, restée malheureusement inachevée, à "la magie». C'est dire l'importance qu'en connaisseur très averti de cette culture il accordait à cet aspect des choses. Hormis dans le texte d'une notice de disque (1966: face A-8 \& 9) : «chants ayant un caractère magique" et "cérémonial magique dont le rythme spécifique se nomme mouya", Simha Arom n'utilise pas (sauf erreur) le terme, auquel il préfere ceux de "conjuratoire » ou de "propitiatoire». De même Michelle Kisliuk, qui elle ne l'utilise jamais (sauf erreur), ce qui est, croirais-je, significatif. Colin Turnbull, après l'avoir utilisé une fois dans la notice de son disque de 1958 (4457 B-8), s'y déclara par la suite (1961), comme il sera dit plus loin, résolument hostile. Que les Pygmées n'aient pas de terme pour désigner ce qu'en ethnologie on nomme magie ne les empêche nullement d'être de fervents magiciens. D'où notre emploi, sans complexe, de ce mot.

14. Deux termes désignent en fait le jodel des Pygmées de l'Ouest. D'une part, comme on vient de le voir, yeli (yeyi, yei) qui désigne le jodel sous sa forme purement chantée, mais plus particulièrement celui des femmes, d'autre part mongombi, qui désigne, stricto sensu, le cri jodlé que poussent les chasseurs partant à la chasse, ou durant la deuxième partie de la chasse au filet pour y rabattre les animaux ainsi piégés, ou encore pour communiquer entre eux en forêt. Mais, dans la pratique, le mot désigne également le chant jodlé des hommes durant une danse quelconque; c'est du moins ce que j'ai cru pouvoir noter en 1946 (et c'est ce qu'indique également Michelle Kisliuk 1998: 52); sur ce jodel, voir aussi infra la note 26. Affaire d'hommes, les femmes ne font pas mongombi. Selon R. F. Thomson qui consacre (1991 : 4752) au jodel de longs commentaires, chez les Pygmées de l'Est, le "code vocal mbuti » comporte la formule "yè $i \grave{e}$ (jodlé) = chante fort ! ", ce qui incite à penser que yeyi, yei, yeli ne sont en fait rien d'autre que des structures syllabiques onomatopéiques et/ou pures porteuses de la vocalisation jodlée. Sur les aspects phonétique et acoustique de la technique du jodel, cf. Suzanne Fürniss (1991). 
15. Djoboko est présenté par Simha Arom (Zoboko 1992: 1 à 3) comme étant, chez les Mbenzélé, "un rituel destiné à apaiser l'esprit d'un éléphant abattu lors d'une grande chasse ". Il constituerait donc chez les BaMbenzélé de la Lobaye l'homologue de Edzingi chez les BaNgombé de la Sangha. Mais, ajoute Arom, il n'est plus aujourd'hui qu'une danse exécutée "pour le simple divertissement", ce qui fut le cas des multiples djoboko enregistrés en 1946 chez les BaMbenzélé de Gatongo. Sur djoboko, voir Simha Arom (1978: disque 1 A-1 à 4), Bahuchet (1985: 433-34: zoboko) et Michelle Kisliuk (1998: 68 : Djoboko).

16. Signalons à ce propos un détail représentatif de la remarquable continuité des représentations symboliques unissant d'un bout à l'autre le discontinuum pygmée : à l'Ouest, un homme, grimpé dans un arbre, "simule la récolte [du miel] en [le] frappant avec sa hache", rapporte Serge Bahuchet (1985: 215), tandis qu’à l'Est, Colin Turnbull (1963: 243) décrit l'«imitation du bruit que font leurs petites haches quand ils s'affairent à la recherche des rayons de miel ». Également représentative, la note suivante.

17. À ces lignes de Colin Turnbull répondent celles de Vincent Dehoux (1995: 78-79) concernant les BaAka de la frontière RCACongo : "l'imitation, par les femmes, du bruit que font les abeilles lorsqu'elles vont butiner est une invite adressée aux hommes pour qu'ils s'adonnent [...] à la recherche des ruches sauvages ».

18. Un enregistrement de sandzo figure dans les deux éditions (1948 et 1957) de la Boîte à Musique. Par suite d'une confusion avec bukela qui lui avait immédiatement succédé ce jour- là, sandzo y est donné pour une danse faisant partie du rituel de chasse. Relues, mes notes disent en fait que sandzo est exécuté "pour le plaisir", d'où ce que j'indique ici. Mais l'affaire est plus compliquée et il me faut entrer dans le détail. Sandzo fut marqué, de manière pour moi très inattendue, par la chute du chef de danse, Mopéta, qui, subitement pris de tremblements, se mit à tituber, puis tomba sans connaissance à terre. Fait évident de transe que j’ai relaté plus longuement qu'ici, en 1980, dans La Musique et la transe, mais dont je n'ai compris que plus tard, à la lecture de Simha Arom, qu'il s'agissait très certainement d'une transe divinatoire. Celle-ci est en rapport avec le feu, ce qui mérite d'être rapproché de ce que relate de son côté Colin Turnbull (1963: 130-132). Reste qu'en
1946 cette chute me fut présentée, de manière catégorique et nonobstant doute et questionnement, comme purement accidentelle ( due au soleil»), et la danse, comme de pur divertissement. Pour le présent, tenons-nous en là.

19. Sur djoboko, voir ci-dessus note 15. Des enregistrements de 1946 en ont été édités dans la série des 78 tours du Musée de l'Homme, et en 1951 chez Columbia par Alan Lomax.

20. Un enregistrement de 1946 en a été publié dans la série des 78 tours du Musée de l'Homme (disque B 24-b), un autre en collaboration avec le Peabody Museum (LD 9 B-2). Michelle Kisliuk (1998: 161) décrit sous le même nom de elanda une danse "incluant hommes, femmes et enfants [dans laquelle] les gens s'avancent au milieu du cercle et font de rapides solos pendant que le groupe chante, mais sans tambours ". Son enregistrement (CD 2-9) montre qu'il s'agit bien de la même musique.

21. Qu'une musique donnée, associée à un rituel précis, soit également exécutée dans un contexte de simple divertissement, Simha Arom (1978) en donne plusieurs exemples. Entre autres: disque 2-A, $6 \& 7$, danse dite Mbenzélé, et disque 3 B-3 où bondo, pièce liée au rituel de divination et donnée spécifiquement ailleurs (1991 : 236) comme réponse aux "désordres", est exécutée "pour le simple plaisir musical». Des faits semblables, signalés en particulier chez les Bochimans, sont, croirais-je, de pratique quasiment universelle (ce qui n'exclut évidemment pas qu'il y ait parfois des interdictions). Avec eux, c'est tout le problème de l'intentionnalité qui est posé.

22. Tripartition sémiologique, selon Jean Molino et Jean-Jacques Nattiez: poïétique/ neutre/ esthésique, ou selon Roman Jakobson : émetteur / message / récepteur - auteurs trop connus pour qu'il soit nécessaire de les référencer. Ici il y a non pas tripartition, mais bipartition, car il n'existe pas de destinataire : il y a le groupe des musiquants, qui ne s'adresse quà lui-même et à personne d'autre. Destinataire, dans le cas de Edzingi celui-ci l'est-il ? Il est participant, il est musiqué, mais aussi chorégraphiant, et donc un récepteur tout à fait différent de celui qui serait spectateur. Il n'y a pas de spectateur. Pour ce qui est de yeli, le destinataire, " esprit » de la forêt, nous met en présence d'une « esthésique » en quelque sorte virtuelle. 
23. Décrit par Vincent Dehoux (1995). Colin Turnbull n'en signale pas l'existence chez les BaMbuti.

24. Chez les BaMbuti de l'Itouri, la longue suite de cérémonies (Colin Turnbull évite le mot rituel) vouées au culte du génie de la forêt nommé Molimo est constamment animée par le jeu de deux longues trompes droites dites, elles aussi, molimo (Turnbull 1963: 64-94). Chez les Bibayak ou Baka du Gabon (Pierre Sallée Face $\mathrm{B} 3 \mathrm{a} \& \mathrm{~b}$ ), une trompe droite, dingolo, intervient constamment dans les «dialogues avec les esprits", parmi lesquels Tsinghi qui "préside à la préparation et à l'issue des grandes chasses à l'éléphant " et n'est autre, note Pierre Sallée, que notre Edzingi des BaNgombé. Ceuxci, autant que j'ai pu le voir en 1946, ne faisaient pas usage de cette trompe. Par ailleurs, l'existence de semblable instrument n'a été mentionnée, sauf erreur, par personne chez les BaAka de la Lobaye, ce qui explique qu'il soit absent de l'article de Suzanne Fürnis et Serge Bahuchet (1995), "Existe-t-il des instruments de musique pygmées?"

25. Le campement de Mopéta, près de Gandicolo, totalisait 34 personnes : 11 hommes dont 2 célibataires et 2 ayant deux femmes, 11 femmes et 12 enfants. Wamata campait à part.

26. Autant chant que cri, c'est le mongombi dont on a parlé ci-dessus à la note 14 . Pour sa part, parlant de ce "qui, pour les Aka, distingue le musical du non-musical», Simha Arom (1991 : 231) écrit que ces «mélopées des chasseurs relèvent pour eux des techniques de la chasse et non pas de la musique». Cf. également Simha Arom (1978, disque 1 A-5). On souhaiterait dès lors savoir en quoi consiste pour eux le concept de musique.

27. « Je n'ai jamais entendu des Pygmées chanter en chœur à l'unisson ", avais-je noté dans ma notice de 1948, ce qui en gros est certes vrai, mais mériterait d'être nuancé. "Horreur de l'unisson [...], redouté autant que les silences ", écrit Simha Arom (1991 : 234).

28. Le fonctionnement de cette polyphonie a été spectaculairement analysé et donné à voir au moyen d'un CD-ROM récemment publié par Simha Arom. La notion de contrepoint y joue un rôle central. Parlant du «flamboiement contrapuntique " propre à cette musique, Pierre Sallée (1981: 45 \& 9) avait proposé d'y voir un étroit rapport avec la technique du jodel. Pour sa part, Yvette Grimaud (1957: 5) avait fait principalement appel, dans son analyse, à la notion de "boucle mélodico-rythmique", boucle où viendrait s'inscrire, entre autres procédés, celui du "contrepoint orné». Personnellement, après avoir repris cette notion de boucle pour caractériser très brièvement, dans un ouvrage de grande diffusion (1963: 212), cette polyphonie, je serais tenté de penser qu'à la notion de contrepoint ("point contre point»), qui implique (Boulez 1958) que «les lignes mélodiques diverses [soient] en relation unique avec [une] ligne principale [le cantus firmus] », pourrait être préférée celle de contreboucle (" boucle contre boucle»), qui rend, me semble-t-il, mieux compte à la fois de cette "mobilité de détail infiniment renouvelée », si justement décrite par Yvette Grimaud, et de cette "impression de perpétuel développement" signalée à son tour par Simha Arom (1991 : 232). Pour d'autres approches de cette polyphonie, voir Suzanne Fürniss (2000) et Fabrice Marandola (2000).

29. Précarité? Du temps où les Pygmées construisaient paisiblement leurs huttes au creux de la forêt, qui n'était en rien pour eux un milieu hostile, Colin Turnbull y insiste beaucoup, ils n'auraient certainement pas qualifié leur situation matérielle de "précaire ", à supposer que le mot ait eu pour eux un sens. Il n'importe. "Quatre ou cinq huttes précaires ", est l'exemple, emprunté à Claudel (parlait-il des Pygmées ?), que donne du mot le Petit Robert.

30. «Être heureux»: les BaMbuti de l'Itouri chantent pour que la forêt "se réveille heureuse ", a-t-on lu plus haut.

31. «Au Congo, les Pygmées victimes du cannibalisme ", titrait en première page Le Monde daté du 27 février 2003.

32. "Espace de survie»: y compris sous les formes les moins attendues. Soit dit sans esprit de provocation, telle a bien été pour eux, quelques soirs de suite, en 1991, la Grande Halle de La Villette, qu’à l'initiative de Michel Boudon un groupe de Pygmées venu de Centrafrique avait investie, et où le public parisien (Danièle Mitterand et Jack Lang notamment) put les voir se produire. Spectacle magnifiquement conçu, sonorisation des chants superbement assurée : on se serait cru en forêt. Reconnaissance exemplaire de cette culture musicale si particulière. Nul doute que pour les Pygmées leur volonté de persévérer de la sorte dans leur être en ait été affer- 
mie. Mais rien n'est simple. Récemment, la tournée en Belgique d'un groupe de Pygmées venus du Cameroun a donné lieu a diverses réactions. "Les Pygmées sont-ils des "bêtes sauvages" ?" titrait Le Monde du 6 septembre 2002, faisant écho à des protestations de "défenseurs des droits de l'homme" et d'"associations africaines", et ajoutant en sous-titre " ... la presse du Cameroun se penche sur leur sort dans leur propre pays... ». D'un côté, donc, de la part de ces "défenseurs", oubli fâcheux que depuis quatre mille ans et le temps des pharaons on sait que les Pygmées sont, dans leur genre, des bateleurs et des intermittents du spectacle; de l'autre, heureuse prise de conscience, chez les Camerounais, du sort qui leur est fait. Musique : espace de survie des Pygmées, mais aussi, pour eux et par contrecoup, espace de résistance.

"Survie ", on s'attendrait à ce que consacrant un article aux «Survival Factors» à l'œuvre chez ces BaMbuti de l'Itouri qu'il a si bien connus et dont il a si bien décrit la musique, Colin Turnbull (1986) cite au moins, à ce propos, leurs activités de musiquants. Aussi surprenant que ce soit, dans cette étude longue de vingt pages il n'a pas un mot pour en parler: elles sont totalement occultées. Aberration conforme à l'orientation générale de l'ouvrage collectif - célèbre (CavalliSforza 1986) - où cet écrit se trouve publié : African Pygmies (son titre) est un exemple d'anthropologie inconsidérément sourde et aveugle à toute une part, essentielle, de la réalité.

33. Alonso Flores, poète et compositeur, qui résida un temps à Nîmes. Citation qui a servi de titre au volume d'hommage à la mémoire de J.-M. Vaccaro. Cf. Franck Dobbins (1998).
34. "Plausible», cf. Jean-Claude Gardin (1987).

35. Ajoutons pour finir que cet article peut être vu (je m'en aperçois après coup) comme se situant dans le prolongement (tardif) d'un débat sur "Comparative Sociomusicology of Classeless and Egalitarian Societies", organisé en 1983 par la Society for Ethnomusicology. Steven Feld y fit longuement référence aux travaux d'Alan Lomax et à son célèbre (et très controversé) Cantometrics (1976), qu'il présenta comme ayant en vue le « musical behavior rather than the musical content" (Feld 1984: 84). Heureuse formule, qui pourrait tout aussi bien convenir au propos présent et qui nous incite à rappeler ici le grand article qu'Alan Lomax publia dans L'Homme (1964), texte très représentatif de tout un aspect de ses recherches. Dans son écrit de 1984, Steven Feld parle surtout, bien entendu, des Kaluli de NouvelleGuinée, et n'y fait qu'une fois mention des Pygmées (1984: 400). Il leur consacre, en revanche, un long article foisonnant, combatif et très bien documenté, dans le Yearbook for Tradional Music (vol. XVIII) qui traite de la très complexe question de l'éthique des publications sonores d'ethnomusicologie. Il y dénonce violemment le sort fait à la fois à la musique pygmée, objet d'une exploitation discographique éhontée, en particulier par le CD Deep Forest, «international disco multi-million seller " (Feld 1996 : 24 et ici même, infra, pp. 396 sq.), et aux Pygmées eux-mêmes, victimes de ce qui s'apparente en fait, me semble-t-il, à un véritable ethnocide. En conclusion des présentes notes, il était indispensable de faire ici mention de ces textes. 
Arom, Simha

1966 Notice pour La musique des Pygmées $B a$-Benzélé (cf. Références discographiques).

1973 Notice pour Aka Pygmy Music (cf. Références discographiques)

1978 Notice pour Anthologie de la musique des Pygmées Aka (cf. Références discographiques).

1983 "Musicologie et ethnomusicologie ", in Encyclopédie des Pygmées Aka. Livre I, fasc. 1 : 29-34 (cf. Jacqueline M. C. Thomas \& Serge Bahuchet).

1991 "La musique omniprésente », in Encyclopédie des Pygmées Aka. Livre I, fasc. 2 : 227-234. (En collaboration avec Vincent Dehoux), (cf. Jacqueline M. C. Thomas \& Serge Bahuchet).

\section{Arom, Simha \& Suzanne Fürniss}

1992 "The Pentatonic System of the Aka Pygmies of the Central African Republic ", in M.-P. Baumann, A. Simon \& U. Wegner, eds, European Studies in Ethnomusicology: Historical Developments and Recent Trends. Selected papers presented at the VII th European Seminar in Ethnomusicology, Berlin, Oct. 1-6, 1990. Wilhelmshafen, Florian Notzel Verlag : 159-173.

\section{Arom, Simha \& Denis-Constant Martin} 1992 Notice pour Polyphonies vocales des Pygmées Benzélé (cf. Références discographiques).

\section{Bahuchet, Serge}

1983 Voir Jacqueline M. C. Thomas.

1985 Les Pygmées Aka et la forêt centrafricaine. Paris, Selaf.

1991 Voir Robert Farris Thompson.

1995a «De la musique considérée comme une philosophie (chez les Pygmées Aka de Centrafrique)", in Ndroje balendro. Musiques, terrains, et disciplines. Textes offerts à Simha Arom. Paris, Éd. Peeters-Selaf : 57-65. 1995b Voir Suzanne Fürniss.
Ballif, Noël

1954 Les Danseurs de Dieu. Chez les Pygmées de la Sangha. Paris, Hachette.

1992 Les Pygmées de la grande forêt. Paris, L'Harmattan.

Bouet, Jacques, Bernard Lortat-Jacob \& Speranta Radulescu

2002 À tue-tête. Chant et violon au Pays de l'Oach, Roumanie. Nanterre, Société d'ethnologie.

Boulez, Pierre

1958 "Contrepoint ", in Encyclopédie de la musique. Paris, Fasquelle.

Brailoiu, Constantin

1949 "À propos du jodel ", KongressBericht, Internationale Gesellschaft für Musikwissenschaft, Basel, 1949. Bâle, Bärenreiter Verlag : 69-71.

\section{Brandel, Rose}

1961 The Music of Central Africa. La Haye, Martinus Nijhoff.

\section{Bundo Daisuke}

2001 "Social Relationship Embodied in Singing and Dancing Performances among the Baka ", African Studies Monographs, Supplementary Issue $n^{\circ} 26: 85-101$.

Cavalli-Sforza, Luigi L., ed.

1986 African Pygmies. Orlando-San DiegoNew York-Austin, Academic Press.

Dehoux, Vincent \& Henri Guillaume 1995 "Chasse, sexualité et musique. Un arc musical des Pygmées Aka ", in Ndroje balendro. Musiques, terrains, et disciplines. Textes offerts à Simha Arom. Paris, Éditions PeetersSelaf: 67-86.

\section{Demesse, Vincent}

1980 Techniques et économie des Pygmées Babinga. Paris, Institut d'ethnologie. 
Dobbins, Franck, ed.

1998 "La musique, de tous les passe-temps le plus beau... ": Hommage à Jean-Michel

Vaccaro. Paris, Klincksieck.

\section{Feld, Steven}

1984 «Sound Structure as Social

Structure " (Symposium on comparative sociomusicology), Ethnomusicology 28 (3) : 383-409.

1996 « Pygmy POP. A Genealogy of Schizophrenic Mimesis ", Yearbook for Traditional Music 28 : 383-409.

\section{Fürniss, Suzanne}

1991 «La technique du jodel chez les Pygmées Aka (Centrafrique). Étude phonétique et acoustique ", Cahiers de musique traditionnelle, "Voix ", 4 : 167-187.

2000 « La conception de la musique vocale chez les Aka : terminologie et combinatoire des paramètres ", Journal des Africanistes 69 (2) : 147-162.

\section{Fürniss, Suzanne \& Serge Bahuchet}

1995 «Existe-t-il des instruments de musique pygmées? ", in Ndroje balendro. Musiques, terrains et disciplines. Textes offerts à Simha Arom. Éditions Peeters-Selaf: 87-109.

\section{Gardin, Jean-Claude}

1987 «Vers une épistémologie pratique en sciences humaines ", in Jean-Claude Gardin et al., eds, La Logique du plausible. Paris, Éditions de la Maison des sciences de l'homme.

Grimaud, Yvette, avec la collaboration de Gilbert Rouget

1957 Notes sur la musique des Bochiman comparée à celle des Pygmées Babinga. Textes (bilingues) et transcriptions musicales pour accompagner le disque Musique bochiman et musique pygmée. Peabody Museum \& Musée de l'Homme (cf. Références discographiques).

\section{Keil, Charles}

1984 "Responses to Feld and Roseman ", Ethnomusicology 28 (3) : 446.
1998 «Call and Response. Applied

Sociomusicology and Performance Studies ", Ethnomusicology 42 (2) : 303-312.

2000 "Compte rendu " de Christofer Small (1998), Ethnomusicology 44 (1) : 161-163.

Kisliuk, Michelle

1998 Seize the Dance! BaAka Musical Life and the Ethnography of Performance. New

York, Oxford University Press [avec 2 CD].

Kubik, Gerhard

2004 Totemismus. Ethnopsychologische

Forschungmaterialien und Interpretationen aus Ost- und Zentralafrika, 1962-2002.

Münster, Lit Verlag. [Le chapitre III (pp. 49-58) est entièrement consacré au Jenge (alias Edzingi) des Pygmées Bangombé de la Haute-Sangha.]

\section{Lévi-Strauss, Claude}

1958 [1949] "L'efficacité symbolique », in Anthropologie structurale. Paris, Plon.

\section{Lomax, Alan}

1964 "Phonotactique du chant populaire", L'Homme 4 (1) : 5-55.

1976 Cantometrics: An Approach to the Anthropology of Music. Berkeley, University of California-Extension Media Center.

\section{Marandola, Fabrice}

2000 "L'apport des nouvelles technologies à l'étude des échelles musicales d'Afrique centrale ", Journal des Africanistes 69 (2): 109-11.

\section{Roche (Dreyfus), Simone}

1952 «Collection Musée de l'Homme (catalogue des disques provenant de la mission Ogooué-Congo) ", in Archives de la musique enregistrée. Paris, UNESCO: 60-66.

\section{Rouget, Gilbert}

1948 "Notice pour les trois disques BAM » $78 \mathrm{tm} / 30 \mathrm{~cm}$ (cf. Références discographiques). 
1949 «Les travaux d'ethnographie musicale de la mission Ogooué-Congo ", Comptes rendus sommaires de l'Institut français d'anthropologie 43 (3) : 4 .

1952 «Notes sur les travaux d'ethnographie musicale de la mission Ogooué-Congo ", Confêrencia Internacional dos Africanistas Occidentais em Bissau, V, 2a parte. Lisboa: 195-204.

1956 Notice pour Musique Pygmée de la Haute-Sangha (cf. Références discographiques).

1957 (ed.) Notes sur la musique des Bochiman comparée à celle des Pygmées Babinga (cf. supra Yvette Grimaud et infra Références discographiques).

1963 "Musique de l'Afrique noire ", in Histoire de la musique I. Paris, Gallimard ("Encyclopédie de la Pléiade ») : 215-237.

1981 (ed.) Courrier du CNRS. Hors série $\mathrm{du} \mathrm{n}^{\circ} 42$ : Ethnomusicologie et représentations de la musique [disque 13/33 encarté].

1990 [1980] La Musique et la transe. Esquisse d'une théorie générale des relations de la musique et de la possession. Préface de Michel Leiris. Paris, Gallimard («Tel»).

\section{Sallée, Pierre}

1975 Notice pour Gabon. Musique des Pygmées Bibayak (cf. Références discographiques).

1981 "Jodel et procédés contrapuntiques des Pygmées ", in Gilbert Rouget, ed., Courrier du CNRS (1981).

Sarno, Louis

1995 Bayaka. The Extraordinary Music of the Babenzele Pygmies. Livre et CD. Cité d'après Michelle Kisliuk 1998.

Schebesta, Paul

1940 Les Pygmées (traduit de l'allemand). Paris, Gallimard.

\section{Small, Christopher}

1996 "Musicking: A Ritual in Social Space », in MUSE (www.musikids.org).
1998 Musicking: The Meanings of Performing and Listening. Hanover, Wesleyan University Press.

Thomas, Jacqueline M. C. \& Serge Bahuchet, eds

1983 Encyclopédie des Pygmées Aka. I : Les Pygmées Aka (fascicule 1). Paris, Lacito-

Selaf.

Thompson, Robert Farris \& Serge Bahuchet 1991 Pygmées? Paris, Musée Dapper.

Tsuru, Daisaku

1998 « Diversity of Ritual Spirit

Performances among the Baka Pygmies of Southeastern Cameroon ", African Studies Monographs, Sup. Issue 25 : 47-84.

Turnbull, Colin

1957-1958 «Notices pour les disques FE 4457 et 4483 ». Rééd. 1991 (cf. Discogr.).

1963 Le Peuple de la forêt. Paris, Stock [Éd. orig. : The Forest People, 1961].

1986 «Survivals Factors among Mbuti and Other Hunters of the Equatorial African

Rain Forest ", in Luigi L. Cavalli-Sforza, ed., African Pygmies : 103-123.

Wallins, Nils L., Björn Merker \& Steven

Brown, eds

2000 The Origins of Music. Cambridge, Mass.-London, The MIT Press.

\section{RÉFÉRENCES DISCOGRAPHIQUES}

\section{Arom, Simha}

1973 Aka Pygmy Music. 1 disque LP 30/33 Philips 6586-016. UNESCO Musical Sources.

1978 Anthologie de la musique des Pygmées Aka (Centrafrique). 3 disques LP 30/33, OCORA 558 526/27/28.

\section{Arom, Simha \& Geneviève Dournon}

1966 La Musique des Pygmées Ba-Benzélé. 1 disque LP 30/33, Bärenreiter Musicaphon BM. 341.2301. Collection UNESCO. 
Arom, Simha \& Denis-Constant Martin

1992 Polyphonies vocales des Pygmées

Benzélé, République centrafricaine. $1 \mathrm{CD}$,

Maison des Cultures du Monde W 260042.

\section{Demolin, Didier}

1991 Pygmées du Haut-Zaïre. Kango, Efe, Asua. CD fmd 190. Musée Dapper.

Kisliuk, Michelle

1998 Seize the Dance! CD 1 \& 2 (Cf. bibliographie).

\section{Lomax, Alan, ed.}

1951 World Library of Folk and Primitive Music, vol. II : French Africa. Columbia Masterworks, 1 LP 30/33, SL-205. Face 2, plage 4 : «Babinga, Bangombe et Babenzele. Cris de chasse, chant magique [yeli], Djoboko, Sandzo, Edjingi ».

\section{Pepper, Herbert}

1958 Anthologie de la vie africaine : MoyenCongo, Gabon. Diverses pièces enregistrées chez les Pygmées BaNgombé et disséminées sur 3 disques LP 30/33, Ducretet-Thomson 320 C 126-127-128.

\section{Rouget, Gilbert}

1947 (M. O-G) )* Music of Equatorial Africa, Ethnic Folkways EFL 1402, Record $1427,78 \mathrm{tm} / 25 \mathrm{~cm}$. Réédité en 1950 (LP), FE 4402 .

1948 (M. O-G) Musiques pygmées et nègres d'Afrique-Équatoriale-Française, 3 disques 30cm/78tm BAM 108-109-110. Rééd. LP 1957.

1948 (M. O-G) Musique de deux groupes de Pygmées, BaBenzélé et BaNgombé, région de Ouesso, ancien Moyen-Congo. Titres: Djoboko, Yéli, Edjingi, Boukéla, Sandzo, Elanda. Série de 10 disques 78 tours ( 6 de $25 \mathrm{~cm}$ et 4 de $30 \mathrm{~cm}$ ). Musée de l'Homme, B 16 à B 25. Ces disques sont présents à la Bibliothèque nationale de France sous les cotes: C010308-312, C010308-312-316 et B001456-459.

1951 (?) (M. O-G) in Alan Lomax, ed., World Library of Folk....
1957 (M. O-G) Musique pygmée de la Haute-Sangha. 1 disque LP, 25/33, La Boîte à musique LD 325, Collection Musée de l'Homme LD 14. Cote de la Bibliothèque nationale de France : E001163.

1957 (ed.) Bushman Music and Pygmy Music = Musique bochiman et musique pygmée. Enregistrements de l'expédition Marsall (1953) et de la Mission Ogooué-Congo (1946). 1 disque 30/33, LD9. Texte de notice par Lorna Marshall. Peabody Museum (Cambridge, Mass.) et Musée de l'Homme (cf. Bibliographie: Yvette Grimaud).

1958 (M.O-G) Chant rituel de chasse.

Collection universelle de musique populaire enregistrée [...]. Brailoiu, ed. UNESCO et Musée d'ethnographie de Genève, disque 3II, 2 (78 tm). Rééd. sur microsillon par les Archives internationales de musique populaire enregistrée, AIMP I B, Genève 1984, Laurent Aubert, ed.

* M. O-G. = Mission Ogooué-Congo 1946. Pour chacun des documents cités lire: "Enregistré par André Didier. Recueilli par Gilbert Rouget ", texte figurant sur la plupart des étiquettes des disques concernés. En effet, durant cette partie de la mission André Didier (alors assistant, plus tard professeur d'enregistrement du son et de l'image au Conservatoire national des Arts et Métiers) enregistrait le son sur disques d'enregistrement direct, opération techniquement délicate, cependant que pour ma part je sélectionnais les musiques à enregistrer et m'occupais des musiciens. Collaboration heureuse s'il en fut et qui se prolongea de même à notre retour, André Didier m’ayant aidé à installer au Département d'ethnologie musicale (tel était alors son nom) du Musée de l'Homme le studio de gravure de disque où furent gravés, de sa propre main, les disques d'enregistrement direct destinés à l'édition entreprise par ce musée. Que ces quelques lignes soient un hommage ému à sa mémoire. Ajoutons, pour la petite histoire (elle le mérite) que les étiquettes de cette édition [34 disques 78 tours/minute, publiés en 50 exemplaires hors commerce, sur des fonds de l'ORSTOM, cf. ci-dessus Simone (Dreyfus) Roche 1952] furent imprimées une à une sur une presse à bras installée au dit Département, par les soins de Lucien Bernot, qui avait été typographe, qui faisait alors ses débuts au Musée de l'Homme, et qui se préparait à partir en Inde à bicyclette; il devait être plus tard professeur au Collège de France. 


\section{Sallée, Pierre}

1975 Gabon. Musique des Pygmées Bibayak. 1 disque LP 30/33. OCORA 558504.

1976 Pygmées et Bochimans. ("Musique \& traditions du monde ", H. de Fraysseix, ed.) 1 disque LP 30/33, CBS 80212.

Turnbull, Colin \& Francis Chapman (M. Kisliuk, ed.)

1991 Mbuti Pygmies of the Ituri Forest. CD SF 40401. Washington, Smithonian

Forlkways. Rééditions de ses anciens LP.
RÉFÉRENCES FILMOGRAPHIQUES

(Mission Ogooué-Congo)

Dupont, Jacques, réalisateur, Edmond Séchan, prise de vue \& P.-D. Gaisseau, assistant

1947 Au pays des Pygmées. Film noir et blanc, $35 \mathrm{~mm}, 24 \mathrm{mn}$. Paris, S.D.A.C. Son post-synchonisé, prise de son : André Didier.

Dupont, Jacques. \& Edmond Séchan

1947 Danses congolaises. Film noir et blanc, $35 \mathrm{~mm},+$ +- $10 \mathrm{mn}$. Paris, S.D.A.C. Son post-synchonisé, prise de son : André Didier.

\section{RÉSUMÉ/ABSTRACT}

Gilbert Rouget, L'efficacité musicale : musiquer pour survivre. Le cas des Pygmées. - Cette recherche se fonde à la fois sur une série de documents musicaux recueillis en $1946 \mathrm{chez}$ les Pygmées BaBinga de la Haute-Sangha (République du Congo) par la mission Ogooué-Congo, et sur l'ensemble des publications ethnomusicologiques parues à ce jour sur les Pygmées, disséminés d'est en ouest de la grande forêt équatoriale, de l'Itouri au Gabon. À la question: "Pourquoi les Pygmées musiquent-ils autant? ", qu'invitent à se poser ces données, il est répondu : "Pour survivre ". "Musiquer" (et non "faire de la musique ") apparaît comme constituant pour eux une véritable technique du corps social et, partant, de la vie collective. Les effets qu'ils en attendent doivent être vus comme conjuguant deux types d'efficacité, d'une part une "efficacité musicale symbolique ", à l'œuvre dans leurs rituels de chasse et liée à leurs pratiques magico-religieuses, d'autre part une "efficacité musicale socio-somatique », à l'œuvre dans l'ensemble des activités musicales (chants et danses) qu'ils mènent quasi quotidiennement. En fin d'article, de nombreuses notes abordent divers problèmes, jodel et polyphonie notamment, concernant cette musique et ce musiquer.
Gilbert Rouget, Music with Effects : "Musicking" to Survive (The Case of the Pygmies). - This research is based on musical documents that the Ogooué-Congo Mission collected in 1946 among the BaBinga Pygmies (HauteSangha, Congo Republic) and on the ethnomusicological publications to date about the Pygmies, who are scattered over the equatorial forest from east to west, from Itouri to Gabon. These documents lead us to ask, "Why do the Pygmies "musick" so much?" and to reply, "To survive." For them, " musicking " - instead of « making music » is a technique of the "social body" and group life. The effects they expect of it must be seen as combining two types of effectiveness: a "symbolic musical effectiveness» operational in their hunting rites and their magic and religious practices; and the "sociosomatic musical effectiveness " of all the musical activities (song and dance) that they do every day. Several notes at the end of the article comment on various problems, in particular yodeling and polyphony. 